\title{
Asset Price Dynamics in a Chartist-Fundamentalist Model with Time Delays: A Bifurcation Analysis
}

\author{
Loretti I. Dobrescu, ${ }^{1}$ Mihaela Neamtu, ${ }^{2}$ and Gabriela Mircea ${ }^{3}$ \\ ${ }^{1}$ School of Economics, University of New South Wales, Sydney, NSW 2052, Australia \\ ${ }^{2}$ Department of Economic Modelling, West University of Timisoara, 16 A J. H. Pestalozzi Street, \\ 300115 Timisoara, Romania \\ ${ }^{3}$ Department of Business Information Systems, West University of Timisoara, 16 A J. H. Pestalozzi Street, \\ 300115 Timisoara, Romania \\ Correspondence should be addressed to Loretti I. Dobrescu; dobrescu@unsw.edu.au
}

Received 25 November 2015; Accepted 11 January 2016

Academic Editor: Zhengqiu Zhang

Copyright ( 2016 Loretti I. Dobrescu et al. This is an open access article distributed under the Creative Commons Attribution License, which permits unrestricted use, distribution, and reproduction in any medium, provided the original work is properly cited.

This paper studies the dynamic behavior of asset prices using a chartist-fundamentalist model with two speculative markets. To this effect, we employ a differential system with delays à la Dibeh (2007) to describe the price dynamics and we assume that the two markets are coupled via diffusive coupling terms. We study two different time delay cases, namely, when both markets experience the same time delay and when the time delay is different across markets. First, we theoretically determine that the equilibrium exists and investigate its stability. Second, we establish the general conditions for the existence of local Hopf bifurcations and analyze their direction and stability. The common conclusion from both the delay scenarios we consider is that coupled speculative markets with heterogeneous agents in each, but with different price dynamics, can be synchronized through diffusive coupling. Finally, we provide some numerical illustrations to confirm our theoretical findings.

\section{Introduction}

When it comes to financial markets dynamics, there is wide consensus that the Efficient Market Hypothesis (EMH) is the standard theory [1]. This theory however fails to explain or predict situations when, for instance, speculative booms are followed by severe crashes. In other words, it cannot explain the excess volatility in these financial markets. As a result, several models have been developed in the last decade to describe markets fluctuations [1-12]. For instance, Dibeh [5] considers a market with two types of participants, namely, fundamentalists and chartists. The first categories of agents follow the EMH theory and base their decisions (and hence their demand formation) on the difference between the actual asset price and the fundamental asset price. On the other hand, the chartists base their market participation decision on the price trend of an asset. Thus, they attempt to exploit past price information when deciding whether to purchase or sell an asset. Specifically, Dibeh [5] used the following delaydifferential equation to describe the dynamics of asset prices:

$$
\begin{aligned}
\dot{p}(t)= & (1-m) \tanh (p(t)-p(t-\tau)) p(t) \\
& -m(p(t)-v) p(t),
\end{aligned}
$$

where $p(t)$ is the actual asset price, $v$ is the fundamental asset price, and $m \in(0,1)$ is the market fraction of fundamentalists. For chartists, the time delay $\tau$ was introduced to capture the fact that they base the slope estimation of their asset price trend on adaptive expectations that consider the past values of the price trend slope. Simulation results showed that there may exist limit cycles for (1), which could explain the persistence of deviations from the fundamental price in speculative markets. These findings are crucial, as Bouchaud and Potters [13] found that, in models considering one asset (i.e., one market), feedback mechanisms are the main driver of market fluctuations, booms, and crashes. 
Using Hassard et al. [14], Qu and Wei [1] provided a theoretical justification for the results in Dibeh [5]. Applying the local Hopf bifurcation theory, they analytically investigated the existence of periodic oscillations for (1), which depends on both the time delay $\tau$ and the market fraction of fundamentalists $m$. Using the normal form theory and center manifold theorem (see also $[15,16]$ ), they derived the sufficient conditions to determine the direction of Hopf bifurcation and the stability of the bifurcating periodic solutions.

In modern finance theory however one of the key ideas is portfolio diversification. And once two or more risky assets (or markets) are available to investors (beliefs on) correlation in price/returns also becomes an investment decision factor. But could agents' beliefs about returns correlation generate comovements in risky assets prices? And how can markets become interdependent? For instance, to what extent may the price dynamics in one market be affected by changes in agents' behavior and beliefs in the alternative market?

In one of the first attempts to tackle these questions, Chiarella et al. [17] developed a discrete time model that combines the chartists-fundamentalists setup with the classical model of diversification between one risk-free and two risky assets. Their analysis showed how chartists' beliefs and behavior may cause increasingly irregular price fluctuations that can be transmitted from one market to another. Furthermore, extending his previous work on models involving one asset (or one market), Dibeh [18] studied the role of feedback mechanism in synchronization and contagion effects (i.e., high asset prices correlation) between different markets. To this effect, the author used a nonlinear chartistfundamentalist model with two markets coupled through a position feedback mechanism given by the price differential between the asset prices.

Drawing on the work in Dibeh [18], in this paper we consider the following system:

$$
\begin{aligned}
\dot{p}_{1}(t)= & \left(1-m_{1}\right) \tanh \left(p_{1}(t)-p_{1}\left(t-\tau_{1}\right)\right) p_{1}(t) \\
& -m_{1}\left(p_{1}(t)-v_{1}\right) p_{1}(t) \\
& +q_{1}\left(p_{2}(t)-p_{1}(t)\right), \\
\dot{p}_{2}(t)= & \left(1-m_{2}\right) \tanh \left(p_{2}(t)-p_{2}\left(t-\tau_{2}\right)\right) p_{2}(t) \\
& -m_{2}\left(p_{2}(t)-v_{2}\right) p_{2}(t) \\
& +q_{2}\left(p_{1}(t)-p_{2}(t)\right),
\end{aligned}
$$

where $m_{i} \in(0,1), i=1,2$, are the market shares of fundamentalists, $\tau_{i} \geq 0, i=1,2$, are time delays, $v_{i}>0$, $i=1,2$, denote the fundamental asset prices, $q_{i} \geq 0, i=1,2$, represent the coupling strength between the two markets, and $I_{t}=p_{1}(t)-p_{2}(t)$ denotes an asset's market index. Note that the expectations function of the chartists is nonlinear, which is conveyed through the hyperbolic tangent function. This formulation represents the chartists' belief that price growth rates are bounded and so it introduces a saturation effect into the chartists demand function. Previous results showed that the synchronization of coupled speculative markets with different dynamics can occur through diffusive coupling. When going from an uncoupled to a coupled market model, the stable converging dynamics is replaced with limit cycle oscillations around the fundamental prices. Thus, coupling and contagion between financial markets can be responsible for the transmission of fluctuations across these markets and appear crucial for their stability [19].

Here is the rationale. The global financial system is formed of a multitude of very diverse markets, located all around the world, and those trade wide-ranging classes of assets. One of their common denominators is that assets price changes often respond to the same economic information and market news [20-22]. This dependency on the same signals leads to price variations that are often correlated. In other words, the price time series can exhibit similar characteristics, which implies that there is "coupling" between markets. Moreover, Fenn et al. [19, 23] found that the strength of correlations between many different assets increases following a credit crisis (i.e., 2007-2008 one), as financial institutions hold similar portfolios of assets [24]. This has important implications for the robustness of financial markets [25]. If many assets are correlated and prices fall, this can cause several financial institutions to write down the value of their assets. And these write-downs can then significantly impact the credit relationships between different institutions [26].

The aim of this paper is to provide a detailed theoretical analysis of the phenomenon of coupling and contagion between markets from the bifurcation point of view. First, we will analytically prove that a model involving two financial markets with correlated prices has equilibrium points that are locally asymptotically stable. Second, for these equilibrium points, we will determine the specific values $\tau_{1}$ and $\tau_{2}$ (i.e., the time delay parameters of the two markets) for which a Hopf bifurcation occurs. Finally, we will study the direction of the Hopf bifurcation, as well as the stability and period of the bifurcating periodic solutions.

We choose the time delays $\tau_{1}$ and $\tau_{2}$ as bifurcation parameters as they represent the "memory" of financial markets. Frank [27] discusses extensively the importance of time delays in modeling financial markets memory and their effect on the qualitative behavior of asset prices. Relatedly, in a 2001 paper, LeBaron [28] also discusses the importance of time horizons in agent-based computational economics. Other works that use time delays as parameters of bifurcations include Hale and Lunel [29], Hassard et al. [14], Mircea et al. [10], Qu and Wei [1], and Xu and Li [30].

The paper proceeds as follows. In Section 2 we determine the equilibrium points of differential system (2) for $v_{1} \neq$ $v_{2}$. Section 3 investigates the local asymptotic stability of the equilibrium points and establishes the existence of the Hopf bifurcation for these points. Section 4 deals with the direction and the stability of the Hopf bifurcation. In Section 5 we use numerical simulations to illustrate the validity of our main results. Section 6 concludes the paper. 


\section{Existence and Stability of the Equilibrium Points}

Consider the model given by system (2). Since time delays do not change, the equilibrium points of this model are given by the solutions of the system:

$$
\begin{aligned}
& m_{1}\left(p_{1}-v_{1}\right) p_{1}-q_{1}\left(p_{2}-p_{1}\right)=0, \\
& m_{2}\left(p_{2}-v_{2}\right) p_{2}-q_{2}\left(p_{1}-p_{2}\right)=0,
\end{aligned}
$$

where $v_{i}>0$ and $m_{i} \in(0,1)$ and $q_{i}>0$, for $i=1,2$. The solution to system (3) is $p_{1}=p_{2}=0$. To find the solution of system (3) with $p_{i}>0$ and $q_{i}>0, i=1,2$, we proceed as follows.

First, from (3) it follows that

$$
\begin{aligned}
& p_{1}=\frac{p_{2}}{q_{2}}\left(q_{2}-m_{2} v_{2}+m_{2} p_{2}\right), \\
& p_{2}=\frac{p_{1}}{q_{1}}\left(q_{1}-m_{1} v_{1}+m_{1} p_{1}\right),
\end{aligned}
$$

which yields

$$
p_{i} \geq v_{i}+\frac{q_{i}}{m_{i}}, \quad i=1,2 .
$$

Since $p_{i}>0$, from (5) it follows that the coupling coefficients $q_{i}, i=1,2$, satisfy the conditions:

$$
q_{i}<m_{i} v_{i}, \quad i=1,2 .
$$

From (4) it follows that $p_{2}$ satisfies the following equation:

$$
a_{3} p_{2}^{3}+a_{2} p_{2}^{2}+a_{1} p_{2}+a_{0}=0,
$$

where

$$
\begin{aligned}
& a_{1}=m_{1}\left(m_{2} v_{2}-q_{2}\right)^{2}-q_{2} m_{2}\left(m_{1} v_{1}-q_{1}\right), \\
& a_{2}=-2 m_{1} m_{2}\left(m_{2} v_{2}-q_{2}\right), \\
& a_{3}=m_{1} m_{2}^{2} \\
& a_{0}=q_{2}\left(m_{2} v_{2}\left(m_{1} v_{1}-q_{1}\right)-q_{2} m_{1} v_{1}\right) .
\end{aligned}
$$

Let

$$
q_{20}=\frac{m_{2} v_{2}\left(m_{1} v_{1}-q_{1}\right)}{\left(m_{1} v_{1}\right)},
$$

and let $q_{21}, q_{22}$ be the roots of

$$
m_{1} x^{2}-m_{2}\left(2 m_{1} v_{2}+m_{1} v_{1}-q_{1}\right) x+m_{1} m_{2}^{2} v_{2}^{2}=0 .
$$

Lemma 1. Let $_{i} \in(0,1), v_{i}>0, i=1,2$, and $q_{1} \in\left(0, m_{1} v_{1}\right)$ is fixed. If

(i) $q_{2} \in\left(0, q_{20}\right)$, then $a_{0}>0, a_{1}>0, a_{2}<0, a_{3}>0$;

(ii) $q_{2} \in\left(q_{20}, q_{21}\right)$, then $a_{0}<0, a_{1}>0, a_{2}<0, a_{3}>0$;

(iii) $q_{2} \in\left(q_{21}, m_{2} v_{2}\right)$, then $a_{0}<0, a_{1}<0, a_{2}<0, a_{3}>0$.
Proof. The proof follows from $a_{3}>0$ and $a_{2}<0$, for any $m_{i} \epsilon$ $(0,1)$ and $v_{i}>0, i=1,2$, and $a_{0}\left(q_{20}\right)=0, a_{1}\left(q_{21}\right)=0$.

Proposition 2. Let

$$
\begin{aligned}
& p=\frac{\left(3 a_{1} a_{3}-a_{2}^{2}\right)}{3 a_{3}^{2}}, \\
& q=\frac{\left(27 a_{0} a_{3}^{2}-9 a_{1} a_{2} a_{3}+2 a_{2}^{3}\right)}{27 a_{3}^{3}}, \\
& \Delta=\frac{q^{2}}{4}+\frac{p^{3}}{27},
\end{aligned}
$$

where $a_{3}, a_{2}, a_{1}$, and $a_{0}$ are given in (8). The equilibrium point of system (2) is $E_{0}\left(p_{10}, p_{20}\right)$, where

$$
\begin{aligned}
& p_{20}=\left(-\frac{q}{2}+\sqrt{\Delta}\right)^{1 / 3}+\left(-\frac{q}{2}-\sqrt{\Delta}\right)^{1 / 3}-\frac{a_{2}}{\left(3 a_{3}\right)}, \\
& p_{10}=\frac{p_{20}\left(m_{2} p_{20}-m_{2} v_{2}+q_{2}\right)}{q_{2}} .
\end{aligned}
$$

For $m_{i} \in(0,1)$ and $v_{i}>0, i=1,2$ fixed, the coordinates of $E_{0}$ are positive.

Proof. The proof follows from Lemma 1 and Cardan's formula, which give the positive solution of a 3 rd-degree equation.

Let $E_{0}\left(p_{10}, p_{20}\right)$ be an equilibrium point, different from $(0,0)$, and let $u_{1}(t)=p_{1}(t)-p_{10}$, and $u_{2}(t)=p_{2}(t)-p_{20}$. System (2) then becomes

$$
\begin{aligned}
& \dot{u}_{1}(t)=a_{11} u_{1}(t)+a_{12} u_{2}(t)+b_{11} u_{1}\left(t-\tau_{1}\right)+F_{1}, \\
& \dot{u}_{2}(t)=a_{21} u_{1}(t)+a_{22} u_{2}(t)+b_{22} u_{2}\left(t-\tau_{2}\right)+F_{2},
\end{aligned}
$$

where

$$
\begin{aligned}
& a_{11}=\left(1-3 m_{1}\right) p_{10}+m_{1} v_{1}-q_{1}, \\
& a_{12}=q_{1}, \\
& b_{11}=-\left(1-m_{1}\right) p_{10}, \\
& a_{21}=q_{2}, \\
& a_{22}=\left(1-3 m_{2}\right) p_{20}+m_{2} v_{2}-q_{2}, \\
& b_{22}=-\left(1-m_{2}\right) p_{20} .
\end{aligned}
$$

The terms $F_{1}, F_{2}$ are given by

$$
\begin{aligned}
F_{1}= & l_{1} u_{1}(t)^{2}+l_{2} u_{1}(t) u_{1}\left(t-\tau_{1}\right)+l_{3} u_{1}(t)^{3} \\
& +l_{4} u_{1}(t)^{2} u_{1}\left(t-\tau_{1}\right)+l_{5} u_{1}(t) u_{1}\left(t-\tau_{1}\right)^{2} \\
& +l_{6} u_{1}\left(t-\tau_{1}\right)^{3}+\text { h.o.t, } \\
F_{2}= & k_{1} u_{2}(t)^{2}+k_{2} u_{2}(t) u_{2}\left(t-\tau_{2}\right)+k_{3} u_{2}(t)^{3} \\
& +k_{4} u_{2}(t)^{2} u_{2}\left(t-\tau_{2}\right)+k_{5} u_{2}(t) u_{2}\left(t-\tau_{2}\right)^{2} \\
& +k_{6} u_{2}\left(t-\tau_{2}\right)^{3}+\text { h.o.t },
\end{aligned}
$$


with $l_{i}, k_{i}, i=1,2$, being given by

$$
\begin{aligned}
& l_{1}=\left(1-2 m_{1}\right) p_{10}, \\
& l_{2}=-\left(1-2 m_{1}\right) p_{10}, \\
& l_{3}=-\left(1-m_{1}\right) \frac{p_{10}}{3}, \\
& l_{4}=\left(1-m_{1}\right) p_{10}, \\
& l_{5}=-\left(1-m_{1}\right) p_{10} \\
& l_{6}=\left(1-m_{1}\right) \frac{p_{10}}{3}, \\
& k_{1}=\left(1-2 m_{2}\right) p_{20}, \\
& k_{2}=-\left(1-2 m_{2}\right) p_{20}, \\
& k_{3}=-\left(1-m_{2}\right) \frac{p_{20}}{3}, \\
& k_{4}=\left(1-m_{2}\right) p_{20}, \\
& k_{5}=-\left(1-m_{2}\right) p_{20}, \\
& k_{6}=\left(1-m_{2}\right) \frac{p_{20}}{3} .
\end{aligned}
$$

The characteristic function for the linear part of (13) is given by

$$
\begin{aligned}
l\left(\lambda, \tau_{1}, \tau_{2}\right)= & \lambda^{2}-\beta_{1} \lambda+\beta_{2}-\left(\beta_{3} \lambda-\beta_{4}\right) e^{-\lambda \tau_{1}} \\
& -\left(\beta_{5} \lambda-\beta_{6}\right) e^{-\lambda \tau_{2}}+\beta_{7} e^{-\lambda\left(\tau_{1}+\tau_{2}\right)},
\end{aligned}
$$

where

$$
\begin{aligned}
& \beta_{1}=a_{11}+a_{22}, \\
& \beta_{2}=a_{11} a_{22}-a_{12} a_{12}, \\
& \beta_{3}=b_{11}, \\
& \beta_{4}=a_{22} b_{11} \\
& \beta_{5}=b_{22} \\
& \beta_{6}=a_{11} b_{22} \\
& \beta_{7}=b_{11} b_{22} .
\end{aligned}
$$

In order to study the distribution of the roots of the transcendental equation $l\left(\lambda, \tau_{1}, \tau_{2}\right)=0$, we use the lemma in Ruan and Wei [31] (see Appendix A) and analyze (17) considering four cases: (i) $\tau_{1}=\tau_{2}=\mathbf{0}$, (ii) $\tau_{1}=\tau_{2}>\mathbf{0}$, (iii) $\tau_{1}>\mathbf{0}, \tau_{2}=\mathbf{0}$, and (iv) $\tau_{1}>\mathbf{0}, \tau_{2}>\mathbf{0}$.

We note that, generally, the analysis of an equation with two delay parameters implies first studying the case when one delay parameter is zero and the other one is different from zero. For this purpose, we set $\tau_{2}=\mathbf{0}$. The next step is to set $\tau_{2}=\mathbf{0}$ as a variable and determine the value (of $\left.\tau_{2}=\mathbf{0}\right)$ for which a Hopf bifurcation occurs. From a practical point of view, only the cases $\tau_{1}=\tau_{2}>\mathbf{0}$ and $\tau_{1}>\mathbf{0}, \tau_{2}>$ $\mathbf{0}$ are relevant. The other two cases (that involve $\tau_{2}=\mathbf{0}$ ) are however needed methodologically, as they allow us to derive some of the results that we need when analyzing the economically relevant cases.

Case 1. If $\tau_{1}=\tau_{2}=\mathbf{0}$, then

$$
l(\lambda, 0,0)=\lambda^{2}-\left(\beta_{1}+\beta_{3}+\beta_{5}\right) \lambda+\beta_{2}+\beta_{4}+\beta_{6}+\beta_{7} .
$$

Using (14) and (18), the equation $l(\lambda, 0,0)=0$ becomes

$$
\begin{aligned}
\lambda^{2}- & \left(2 m_{1} p_{10}+2 m_{2} p_{20}-\left(m_{1}+m_{2}\right) v_{2}+q_{1}+q_{2}\right) \lambda \\
& +\left(4 m_{1} m_{2}+1\right) p_{10} p_{20}-2 m_{1}\left(m_{2} v_{2}-q_{2}\right) p_{10} \\
& -2 m_{2}\left(m_{1} v_{1}-q_{1}\right)-q_{1} q_{2}=0 .
\end{aligned}
$$

The necessary and sufficient conditions for (20) to admit roots with a negative real part are

$$
\begin{aligned}
& \left(2 m_{1} p_{10}-4 m_{1} m_{2}+1\right) p_{10} p_{20}-2 m_{1}\left(m_{2} v_{2}-q_{2}\right) p_{10} \\
& \quad-2 m_{2}\left(m_{1} v_{1}-q_{1}\right)-q_{1} q_{2}>0, \\
& 2 m_{1} p_{10}+2 p_{10}-\left(m_{1}+m_{2}\right) v_{2}+q_{1}+q_{2}<0 .
\end{aligned}
$$

If the conditions in (21) hold, then the equilibrium point $E_{0}\left(p_{10}, p_{20}\right)$ is locally asymptotically stable.

\section{The Local Stability of the Hopf Bifurcation}

In the analysis of the Hopf bifurcation, we consider $\tau_{1}$ and $\tau_{2}$ as parameters and $E_{0}\left(p_{10}, p_{20}\right)$ as an equilibrium point for which one of the situations presented in Proposition 2 is verified.

Case 2. If $\tau_{1}=\tau_{2}=\tau>\mathbf{0}$, the characteristic function (17) becomes

$$
\begin{aligned}
l(\lambda, \tau, \tau)= & \lambda^{2}-\beta_{1} \lambda+\beta_{2}-\left(\beta_{8} \lambda-\beta_{9}\right) e^{-\lambda \tau} \\
& +\beta_{7} e^{-2 \lambda \tau},
\end{aligned}
$$

where

$$
\begin{aligned}
& \beta_{8}=\beta_{3}+\beta_{5}, \\
& \beta_{9}=\beta_{4}+\beta_{6} .
\end{aligned}
$$

We now rewrite (22) as

$$
e^{\lambda \tau}\left(\lambda^{2}-\beta_{1} \lambda+\beta_{2}\right)-\beta_{8} \lambda+\beta_{9}+\beta_{7} e^{-\lambda \tau}=0,
$$

noting that when $\tau>0, \lambda=i \omega(\omega>0)$ is a root of (24) if and only if $\omega$ satisfies

$$
\begin{aligned}
& \left(\beta_{2}+\beta_{7}-\omega^{2}\right) \cos (\omega \tau)+\beta_{1} \omega \sin (\omega \tau)=-\beta_{9}, \\
& \left(\beta_{2}-\beta_{7}-\omega^{2}\right) \sin (\omega \tau)-\beta_{1} \omega \cos (\omega \tau)=\beta_{8} \omega .
\end{aligned}
$$

By direct computation, we have

$$
\begin{aligned}
& \cos (\omega \tau)=\frac{\beta_{9}\left(\omega^{2}+\beta_{7}-\beta_{2}\right)-\beta_{1} \beta_{8} \omega^{2}}{\left(\beta_{2}+\beta_{7}-\omega^{2}\right)\left(\beta_{2}-\beta_{7}-\omega^{2}\right)+\beta_{1}^{2} \omega^{2}}, \\
& \sin (\omega \tau)=\frac{\beta_{8} \omega\left(\beta_{2}+\beta_{7}-\omega^{2}\right)-\beta_{1} \beta_{9} \omega}{\left(\beta_{2}+\beta_{7}-\omega^{2}\right)\left(\beta_{2}-\beta_{7}-\omega^{2}\right)+\beta_{1}^{2} \omega^{2}} .
\end{aligned}
$$


Using $\cos (\omega \tau)^{2}+\sin (\omega \tau)^{2}=1$, from (26), it follows that

$$
\begin{aligned}
& \omega^{8}+\gamma_{6} \omega^{6}+\gamma_{4} \omega^{4}+\gamma_{2} \omega^{2}+\gamma_{0}=0, \\
& \gamma_{6}=2\left(\beta_{1}^{2}-2 \beta_{2}\right)-\beta_{8}^{2},
\end{aligned}
$$

$\gamma_{4}$

$$
\begin{aligned}
= & \left(\beta_{1}^{2}-2 \beta_{2}\right)^{2}+2\left(\beta_{2}^{2}-\beta_{7}^{2}\right) \\
& +2 \beta_{8}\left(\beta_{8}\left(\beta_{2}+\beta_{7}\right)-\beta_{1} \beta_{9}\right)-\left(\beta_{9}-\beta_{1} \beta_{8}\right)^{2},
\end{aligned}
$$

$\gamma_{2}$

$$
\begin{aligned}
= & 2\left(\beta_{1}^{2}-2 \beta_{2}\right)\left(\beta_{2}^{2}-\beta_{7}^{2}\right) \\
& -2\left(\beta_{9}-\beta_{1} \beta_{8}\right)\left(\beta_{7}-\beta_{2}\right) \beta_{9} \\
& -\left(\beta_{8}\left(\beta_{2}+\beta_{7}\right)-\beta_{1} \beta_{9}\right)^{2},
\end{aligned}
$$

$\gamma_{0}=\beta_{2}^{2}-\beta_{7}^{2}-\beta_{9}\left(\beta_{7}-\beta_{2}\right)^{2}$.

Denoting $z=\omega^{2}$, (27) yields

$$
z^{4}+\gamma_{6} z^{3}+\gamma_{4} z^{2}+\gamma_{2} z+\gamma_{0}=0
$$

Let

$$
h(z)=z^{4}+\gamma_{6} z^{3}+\gamma_{4} z^{2}+\gamma_{2} z+\gamma_{0}
$$

and define

$$
\begin{aligned}
p & =\frac{8 \gamma_{4}-3 \gamma_{6}^{2}}{16}, \\
q & =\frac{\gamma_{6}^{3}-4 \gamma_{4} \gamma_{6}+8 \gamma_{2}}{32}, \\
D_{0} & =\frac{q^{2}}{4}+\frac{p^{3}}{27} .
\end{aligned}
$$

Then, from the sign of $D_{0}$, we have that

$$
\begin{aligned}
& z_{10}=-\frac{\gamma_{6}}{4}+\sqrt[3]{-\frac{q}{2}+\sqrt{D_{0}}}+\sqrt[3]{-\frac{q}{2}-\sqrt{D_{0}}} \\
& \text { if } D_{0}>0 \text {, } \\
& z_{20}=\max \left\{-\frac{\gamma_{6}}{4}+\sqrt[3]{-\frac{q}{2}},-\frac{\gamma_{6}}{4}-\sqrt[3]{-\frac{q}{2}}\right\}, \quad \text { if } D_{0}=0 \text {, } \\
& z_{30}=\max \left\{r_{10}, r_{20}, r_{30}\right\}, \quad \text { if } D_{0}<0 \text {, }
\end{aligned}
$$

$$
\tau_{j}=\frac{1}{\omega_{0}}\left[\arccos \left(\frac{\Delta_{1}}{\Delta}\right)+2 j \pi\right], \quad j=0,1,2, \ldots,
$$

where

$$
\begin{aligned}
\Delta_{1} & =\beta_{9}\left(\omega_{0}^{2}+\beta_{7}-\beta_{2}\right)-\beta_{1} \beta_{8} \omega_{0}^{2}, \\
\Delta & =\left(\beta_{2}+\beta_{7}-\omega_{0}^{2}\right)\left(\beta_{2}-\beta_{7}-\omega_{0}^{2}\right)+\beta_{1}^{2} \omega_{0}^{2} .
\end{aligned}
$$

From the above relations, we have the following result.

Proposition 3. Let $\beta_{i}, i=1, \ldots, 7, \gamma_{6}, \gamma_{4}, \gamma_{2}, \gamma_{0}$, and $\tau_{j}$ be given by (18), (28), and (36). Suppose that conditions (34) are satisfied and that the positive root of (29) satisfies $h^{\prime}\left(z_{0}\right) \neq$ 0 . Then, the equilibrium point $E_{0}\left(p_{10}, p_{20}\right)$ is asymptotically stable, when $\tau \in\left[0, \tau_{0}\right)$, and it is unstable, when $\tau>$ $\tau_{0}$. Moreover, at $\tau=\tau_{j}, j=0,1,2, \ldots, \pm i \omega_{0}$ is a pair of simple imaginary roots of (29) and system (2) undergoes Hopf bifurcation near $E_{0}\left(p_{10}, p_{20}\right)$. If (29) does not have a positive root, then the equilibrium point $E_{0}\left(p_{10}, p_{20}\right)$ is locally asymptotically stable for all $\tau \geq 0$.

Case 3. If $\tau_{1}>\mathbf{0}$ and $\tau_{2}=\mathbf{0}$, then $l\left(\lambda, \tau_{1}, 0\right)=0$ becomes

$$
\begin{aligned}
\lambda^{2} & -\left(\beta_{1}+\beta_{5}\right) \lambda+\beta_{2}+\beta_{6}-\left(\beta_{3} \lambda-\beta_{4}-\beta_{7}\right) e^{-\lambda \tau_{1}} \\
& =0 .
\end{aligned}
$$

For $\omega>0$, let $\lambda=i \omega$ be a root of (37). It then follows that

$$
\begin{aligned}
& \left(\beta_{4}+\beta_{7}\right) \cos \left(\omega \tau_{1}\right)-\beta_{3} \omega \sin \left(\omega \tau_{1}\right)=\omega^{2}-\beta_{2}-\beta_{6}, \\
& \left(\beta_{4}+\beta_{7}\right) \sin \left(\omega \tau_{1}\right)+\beta_{3} \omega \cos \left(\omega \tau_{1}\right)=-\left(\beta_{1}+\beta_{5}\right) \omega,
\end{aligned}
$$

which leads to

$$
\begin{aligned}
\omega^{4}+\delta_{2} \omega^{2}+\delta_{0} & =0, \\
\delta_{2} & =\left(\beta_{1}+\beta_{5}\right)^{2}-2\left(\beta_{2}+\beta_{6}\right)-\beta_{3}^{2}, \\
\delta_{0} & =\left(\beta_{2}+\beta_{6}\right)^{2}-\left(\beta_{4}+\beta_{7}\right)^{2} .
\end{aligned}
$$

It is easy to see that if the conditions

$$
\begin{aligned}
& \delta_{2}>0, \\
& \delta_{0}>0
\end{aligned}
$$

hold, then (39) has no positive roots. Hence, all the roots of (37) have negative real parts when $\tau_{1} \in[0, \infty]$.

If $\delta_{0}<0$, then (39) has a unique positive root $\omega_{10}^{2}$. Substituting $\omega_{10}^{2}$ into (38), we obtain and $\alpha$ is one of the cubic roots of the complex number $-q / 2+$ $\sqrt{D_{0}}$, and $\varepsilon=-1 / 2+i(\sqrt{3} / 2)$. 


$$
\tau_{1 j}=\frac{\left[\arccos \left(\left(\left(\omega_{10}^{2}-\beta_{2}-\beta_{6}\right)\left(\beta_{2}+\beta_{7}\right)-\beta_{3}\left(\beta_{1}+\beta_{5}\right) \omega_{10}^{2}\right) /\left(\left(\beta_{1}+\beta_{7}\right)^{2}+\beta_{3} \omega_{10}^{2}\right)\right)+2 j \pi\right]}{\omega_{10}},
$$

with $j=0,1,2, \ldots$ If the conditions

$$
\begin{aligned}
& \delta_{2}<0 \\
& \delta_{0}>0
\end{aligned}
$$$$
\tau_{1 k \pm}=\frac{\left[\arccos \left(\left(\left(\omega_{10 \pm}^{2}-\beta_{2}-\beta_{0}\right)\left(\beta_{2}+\beta_{7}\right)-\beta_{3}\left(\beta_{1}+\beta_{5}\right) \omega_{10 \pm}^{2}\right) /\left(\left(\beta_{1}+\beta_{7}\right)^{2}+\beta_{3} \omega_{10 \pm}^{2}\right)\right)+2 k \pi\right]}{\omega_{10 \pm}},
$$

hold, then (39) has two positive roots $\omega_{10+}^{2}$ and $\omega_{10-}^{2}$. Substituting $\omega_{10 \pm}^{2}$ into (38), we obtain with $k=0,1,2, \ldots$

Let $\lambda\left(\tau_{1}\right)=\alpha\left(\tau_{1}\right)+i \omega\left(\tau_{1}\right)$ be a root of (37) near $\tau_{1}=\tau_{1 n},(n=0,1,2, \ldots)$ and $\alpha\left(\tau_{1 n}\right)=0, \omega\left(\tau_{1 n}\right)=\omega_{0}$. According to the functional differential equation theory, for every $\tau_{1 n}(n=0,1,2, \ldots)$, there exists $\varepsilon>0$ such that $\lambda\left(\tau_{1}\right)$ is continuously differentiable in $\tau_{1}$ for $\left|\tau_{1}-\tau_{1 n}\right|<\varepsilon$. Substituting $\lambda\left(\tau_{1}\right)$ into the LHS of (37) and taking derivative with respect to $\tau_{1}$ yield

$$
\left(\frac{d \lambda\left(\tau_{1}\right)}{d \tau_{1}}\right)^{-1}=\frac{-\left(2 \lambda-\beta_{1}-\beta_{5}\right) e^{\lambda \tau_{1}}+\beta_{3}}{\lambda\left(\beta_{3} \lambda-\beta_{4}-\beta_{7}\right)}-\frac{\tau_{1}}{\lambda}
$$

which leads to

$$
\left(\frac{d \operatorname{Re}\left(\lambda\left(\tau_{1}\right)\right)}{d \tau_{1}}\right)_{\tau_{1}=\tau_{1 n}}^{-1}=\sqrt{\delta_{2}^{2}-4 \delta_{0}}
$$

Since $\operatorname{sign}\left(d \operatorname{Re}\left(\lambda\left(\tau_{1}\right)\right) / d \tau_{1}\right)_{\tau_{1}=\tau_{1 n}}^{-1} \quad=\quad \operatorname{sign}\left(\operatorname{Re}\left(d \lambda\left(\tau_{1}\right) /\right.\right.$ $\left.\left.d \tau_{1}\right)\right)_{\tau_{1}=\tau_{1 n}}^{-1}$, we have that

$$
\left.\frac{d \operatorname{Re}\left(\lambda\left(\tau_{1}\right)\right)}{d \tau_{1}}\right|_{\tau_{1}=\tau_{1 n}}>0 .
$$

Similarly, we can obtain

$$
\begin{aligned}
& \left.\frac{d \operatorname{Re}\left(\lambda\left(\tau_{1}\right)\right)}{d \tau_{1}}\right|_{\tau_{1}=\tau_{1 k+}}>0, \\
& \left.\frac{d \operatorname{Re}\left(\lambda\left(\tau_{1}\right)\right)}{d \tau_{1}}\right|_{\tau_{1}=\tau_{1 k-}}<0 .
\end{aligned}
$$

From the above analysis and Ruan and Wei [31, Corollary 2.4], we have the following result.

Proposition 4. For $\tau_{2}=0$, assume that either condition (41) or condition (43) is satisfied. Then, the following results hold.

(i) If (41) holds, the equilibrium point $E_{0}\left(p_{10}, p_{20}\right)$ is asymptotically stable for all $\tau_{1}>0$. (ii) If (43) holds, then there is a positive integer $m$ such that the equilibrium point $E_{0}\left(p_{10}, p_{20}\right)$ is asymptotically stable when $\tau_{1} \in\left[0, \tau_{10+}\right] \cup\left[\tau_{10-}, \tau_{11+}\right] \cup$ $\cdots \cup\left[\tau_{1 m-1-}, \tau_{1 m+}\right]$, and it is unstable when $\tau_{1} \in$ $\left[\tau_{10+}, \tau_{10-}\right] \cup\left[\tau_{11+}, \tau_{11-}\right] \cup \cdots \cup\left[\tau_{1 m+}, \tau_{1 m-}\right] \cup\left[\tau_{1 m+}, \infty\right]$. Moreover, at $\tau_{1}=\tau_{1 k_{ \pm}}, k=0,1,2, \ldots$, system (2) undergoes Hopf bifurcation at $E_{0}\left(p_{10}, p_{20}\right)$, where $\tau_{1 k \pm}$ are given by (44).

Case 4. Let $\tau_{1}>\mathbf{0}$ and $\tau_{2}>\mathbf{0}$. We consider (17), with $\tau_{1}$ in its stable interval $\left[0, \tau_{10}\right]$, and regard $\tau_{2}$ as a parameter. Without loss of generality, we assume that $\delta_{0}<0$ and let $\lambda=i \omega(\omega>$ 0 ) be a root of (17). Then, we can obtain

$$
\begin{aligned}
& \left(\beta_{6}+\beta_{7} \cos \left(\omega \tau_{1}\right)\right) \cos \left(\omega \tau_{2}\right) \\
& -\left(\beta_{5} \omega+\beta_{7} \sin \left(\omega \tau_{1}\right)\right) \sin \left(\omega \tau_{2}\right)=\omega^{2}-\beta_{2} \\
& \quad-\beta_{4} \cos \left(\omega \tau_{1}\right)+\beta_{3} \omega \sin \left(\omega \tau_{1}\right), \\
& \left(\beta_{5} \omega+\beta_{7} \sin \left(\omega \tau_{1}\right)\right) \cos \left(\omega \tau_{2}\right) \\
& \quad+\left(\beta_{6}+\beta_{7} \cos \left(\omega \tau_{1}\right)\right) \sin \left(\omega \tau_{2}\right)=-\beta_{1} \omega \\
& -\beta_{3} \omega \cos \left(\omega \tau_{1}\right)-\beta_{4} \sin \left(\omega \tau_{1}\right),
\end{aligned}
$$

which leads to

$$
\omega^{4}+\varepsilon_{3} \omega^{3}+\varepsilon_{2} \omega^{2}+\varepsilon_{1} \omega+\varepsilon_{0}=0,
$$

where

$$
\begin{aligned}
& \varepsilon_{3}(\omega)=2 \beta_{3} \sin \left(\omega \tau_{1}\right) \\
& \varepsilon_{2}(\omega)=\beta_{3}^{2}-2 \beta_{2}-2\left(\beta_{4}-\beta_{1} \beta_{3}\right) \cos \left(\omega \tau_{1}\right), \\
& \varepsilon_{1}(\omega)=\left(2 \beta_{1} \beta_{4}-2 \beta_{2} \beta_{3}\right) \sin \left(\omega \tau_{1}\right) \\
& \varepsilon_{0}(\omega)=\left(\beta_{2}^{2}+\beta_{4}^{2}+2 \beta_{1} \beta_{4}\right) \cos \left(\omega \tau_{1}\right)
\end{aligned}
$$

Denote

$$
\begin{aligned}
K_{21}(\omega)= & \omega^{4}+\varepsilon_{3}(\omega) \omega^{3}+\varepsilon_{2}(\omega) \omega^{2}+\varepsilon_{1}(\omega) \omega \\
& +\varepsilon_{0}(\omega) .
\end{aligned}
$$


We assume that there exists $\omega_{0}>p$ such that $K_{21}\left(\omega_{0}\right)=0$. For $\omega_{0}$, there exists a sequence $\left\{\tau_{2 j}, j=1,2, \ldots\right\}$ such that (50) holds.

Let $\tau_{21}=\max \left\{\tau_{2 i}, i=1, \ldots\right\}$. When $\tau_{2}=\tau_{21}$, (17) has a pair of purely imaginary roots $\pm i \omega_{21}$, for $\tau_{1} \in\left[0, \tau_{10}\right)$.

In the following we assume that

$$
\left(\frac{d \operatorname{Re}(\lambda(\tau))}{d \tau_{2}}\right)_{\lambda=i \omega_{21}}^{-1} \neq 0
$$

Thus, by the general Hopf bifurcation theorem for FDEs in Hale and Lunel [29], we have the following result on stability and Hopf bifurcation for system (2).

Proposition 5. Assume that the above condition, $\left(d \operatorname{Re}(\lambda(\tau)) / d \tau_{2}\right)_{\lambda=i \omega_{21}}^{-1} \quad \neq \quad 0$, is satisfied and that $\tau_{1} \in\left[0, \tau_{10}\right)$. Then, the equilibrium point $E_{0}\left(p_{10}, p_{20}\right)$ is locally asymptotically stable when $\tau_{2} \in\left[0, \tau_{21}\right)$. Moreover, when $\tau_{2}=\tau_{21}$, system (2) undergoes Hopf bifurcation at $E_{0}\left(p_{10}, p_{20}\right)$.

If $\omega_{21}$ is a positive root of (50), then $\tau_{2 j}$ is given by

$$
\tau_{2 j}=\frac{1}{\omega_{21}}\left[\arccos \left(\frac{H_{21}}{L_{21}}\right)+2 j \pi\right], \quad j=0,1,2, \ldots,
$$

where

$$
\begin{aligned}
& H_{21} \\
& \quad=\left(\omega_{21}^{2}-\beta_{2}-\beta_{1} \cos \left(\omega_{21} \tau_{1}\right)+\beta_{3} \omega_{21} \sin \left(\omega_{21} \tau_{1}\right)\right) \\
& \cdot\left(\beta_{6}+\beta_{7} \cos \left(\omega_{21} \tau_{1}\right)\right) \\
& \quad-\left(\omega_{21} \beta_{1}+\beta_{3} \omega_{21} \cos \left(\omega_{21} \tau_{1}\right)+\beta_{1} \sin \left(\omega_{21} \tau_{1}\right)\right) \\
& \quad \cdot\left(\beta_{2} \omega_{21}+\beta_{7} \sin \left(\omega_{21} \tau_{1}\right)\right) \\
& L_{21}=\left(\beta_{6}+\beta_{7} \cos \left(\omega_{21} \tau_{1}\right)\right)^{2} \\
& \quad+\left(\beta_{3} \omega_{21}+\beta_{7} \sin \left(\omega_{21} \tau_{1}\right)\right)^{2} .
\end{aligned}
$$

\section{The Direction and the Stability of the Hopf Bifurcation}

In the previous section, we have obtained the condition for the Hopf bifurcation to occur when $\tau_{1}=\tau_{10}$, where $\tau_{10}$ is given by (42). Using the techniques from normal form and center manifold theory introduced by Hassard et al. [14], we now derive the explicit formulae determining the direction, stability, and periodicity of these solutions bifurcating from the positive equilibrium $E_{0}\left(p_{10}, p_{20}\right)$ at the critical value $\tau_{1}=$ $\tau_{10}$. To this effect, we will use the first Lyapunov coefficient.

Throughout this section, we assume that differential system (2) undergoes a Hopf bifurcation at the positive equilibrium $E_{0}\left(p_{10}, p_{20}\right)$ for $\tau_{1}=\tau_{10}$ and that $\pm i \omega_{10}$ are the corresponding purely imaginary roots of the characteristic equation at $E_{0}\left(p_{10}, p_{20}\right)$. Without loss of generality, we assume that $\tau_{2}<\tau_{10}$, where $\tau_{2} \in\left(0, \tau_{21}\right)$ and $\tau_{21}$ is given in (54). For convenience, let $\bar{u}_{i}(t)=u_{i}\left(\tau_{1} t\right), i=1,2$, and $\tau_{1}=\tau_{10}+\mu$, where $\tau_{10}$ is given by (42) and $\mu \in \mathbb{R}$. Differential system (2) can be written as a FDE in $C=C\left([-1,0], \mathbb{R}^{2}\right)$ as follows:

$$
\dot{u}(t)=L_{\mu}\left(u_{t}\right)+F\left(\mu, u_{t}\right),
$$

where $u(t)=\left(u_{1}(t), u_{2}(t)\right)^{T} \in C$ and $u_{t}(\phi)=u(t+\theta)=$ $\left(u_{1}(t+\theta), u_{2}(t+\theta)\right)^{T} \in C$. The terms $L_{\mu}: C \rightarrow \mathbb{R}$ and $F: \mathbb{R} \times C \rightarrow \mathbb{R}$ are, respectively, given by

$$
\begin{aligned}
L_{\mu} \phi & =A \phi(0)+B_{1} \phi(-1)+B_{2} \phi\left(-\frac{\tau_{2}}{\tau_{10}}\right), \\
F(\mu, \phi) & =\left(F_{11}(\phi), F_{12}(\phi)\right)^{T},
\end{aligned}
$$

where $\phi(\theta)=\left(\phi_{1}(\theta), \phi_{2}(\theta)\right)^{T} \in C$ and

$$
\begin{aligned}
A & =\left(\begin{array}{ll}
a_{11} & a_{12} \\
a_{21} & a_{22}
\end{array}\right), \\
B_{1} & =\left(\begin{array}{ll}
b_{11} & 0 \\
0 & 0
\end{array}\right), \\
B_{2} & =\left(\begin{array}{ll}
0 & 0 \\
0 & b_{22}
\end{array}\right), \\
F_{11}(\phi) & =\left(\tau_{10}+\mu\right) F_{1}\left(\phi_{1}(0), \phi_{1}(-1)\right), \\
F_{12}(\phi) & =\left(\tau_{10}+\mu\right) F_{2}\left(\phi_{2}(0), \phi_{2}\left(-\frac{\tau_{2}}{\tau_{10}}\right)\right),
\end{aligned}
$$

with $F_{1}$ and $F_{2}$ being given by (13).

From Section 3 we know that if $\mu=0$, then differential system (2) undergoes a Hopf bifurcation at $E_{0}\left(p_{10}, p_{20}\right)$ and the characteristic equation associated with system (2) has a pair of simple imaginary roots $\pm i \omega_{10} \tau_{10}$. By the Reisz representation theorem, there is a matrix function with bounded variation components $\eta(\theta, \mu), \theta \in[-1,0]$ such that

$$
L_{\mu} \phi=\int_{-1}^{0} d \eta(\theta, \mu) \phi(\theta), \quad \text { for } \phi \in C .
$$

We can choose

$$
\eta(\theta, \mu)= \begin{cases}A_{1}+B_{11}+B_{12}, & \theta=0 \\ B_{11}+B_{12}, & \theta \in\left[-\frac{\tau_{2}}{\tau_{10}}, 0\right) \\ B_{11}, & \theta \in\left(-1,-\frac{\tau_{2}}{\tau_{10}}\right) \\ 0, & \theta=-1 .\end{cases}
$$

For $\phi \in C\left([-1,0], \mathbb{R}^{3}\right)$, we define

$$
\begin{aligned}
A(\mu) \phi(\theta) & = \begin{cases}\frac{d \phi(\theta)}{d \theta}, & \theta \in[-1,0) \\
\int_{-1}^{0} d \eta(s, \mu) \phi(s), & \theta=0,\end{cases} \\
R\left(\mu_{2}\right) \phi & = \begin{cases}0, & \theta \in[-1,0) \\
F(\mu, \phi), & \theta=0 .\end{cases}
\end{aligned}
$$


Then, (57) is equivalent to the abstract differential equation:

$$
\dot{u}_{t}(t)=A(\mu) u_{t}+R(\mu) u_{t}
$$

where $u_{t}=u(t+\theta)$, for $\theta \in[-1,0]$.

For $\phi \in C\left([-1,0],\left(\mathbb{R}^{2}\right)^{*}\right)$, we define

$$
A^{*} \psi(s)= \begin{cases}\frac{-d \psi(s)}{d s}, & s \in(0,1] \\ \int_{-1}^{0} d \eta^{T}(t, 0) \psi(s), & s=0 .\end{cases}
$$

For $\phi \in C\left([-1,0], \mathbb{R}^{2}\right)$ and $\psi \in C\left([0,1],\left(\mathbb{R}^{2}\right)^{*}\right)$, we define the bilinear form

$$
\begin{aligned}
\langle\psi, \phi\rangle= & \bar{\psi}(0) \phi(0) \\
& -\int_{-1}^{0} \int_{\xi=0}^{\theta} \psi^{T}(\xi-\theta) d \eta(\theta) \phi(\xi) d \xi,
\end{aligned}
$$

where $\eta(\theta)=\eta(\theta, 0), A=A(0)$, and $A^{*}$ are adjoint operators. Following from Section 3 discussion, we also know that $\pm i \omega_{10} \tau_{10}$ are eigenvalues of $A(0)$, but they are also eigenvalues of $A^{*}$.

Proposition 6. (i) If the eigenvector $q$ of $A(\mu)$ is given by

$$
\begin{aligned}
q(\theta) & =(1, \alpha)^{T} e^{i \omega_{10} \tau_{10} \theta}, \quad \theta \in(-1,0], \text { then } \\
\alpha & =\frac{i \omega_{10} \tau_{10}-a_{11}-b_{11} e^{-i \omega_{10} \tau_{10}}}{a_{12}} .
\end{aligned}
$$

(ii) If the eigenvector $q^{*}$ of $A^{*}$ is given by

$$
\begin{aligned}
q^{*}(s) & =\frac{1}{\rho}\left(1, \alpha^{*}\right)^{T} e^{i \omega_{10} \tau_{10} s}, \quad s \in[0,1), \text { then } \\
\alpha^{*} & =-\frac{i \omega_{10} \tau_{10}+a_{11}+b_{11} e^{-i \omega_{10} \tau_{10}}}{a_{21}} .
\end{aligned}
$$

The proof is provided in Appendix A.

Using $\left\langle q, q^{*}\right\rangle=1$, we can obtain $\rho$ as follows:

$$
\begin{aligned}
& \left\langle q, q^{*}\right\rangle \\
& =\bar{q}^{*}(0)^{T} q(0) \\
& \quad-\int_{\theta=-1}^{0} \int_{\xi=0}^{\theta} \bar{q}^{* T}(\xi-\theta) d \eta(\theta) q(\xi) d \xi \\
& =\frac{1}{\rho}\left(1+\alpha \bar{\alpha}^{*}\right) \\
& \quad+\frac{1}{\rho}\left[\tau_{10} b_{11} e^{-i \omega_{10} \tau_{10}}+\frac{\tau_{2}}{\tau_{10}} b_{22} \alpha \bar{\alpha}^{*} e^{-i \omega_{10} \tau_{2} / \tau_{10}}\right] \\
& =1 .
\end{aligned}
$$

Hence, we have

$$
\rho=1+\alpha \bar{\alpha}^{*}+\tau_{10} b_{11} e^{-i \omega_{10} \tau_{10}}+\frac{\tau_{2}}{\tau_{10}} b_{22} \alpha \bar{\alpha}^{*} e^{-i \omega_{10} \tau_{2} / \tau_{10}} .
$$

Similarly, we can verify that $\left\langle q^{*}(s), \bar{q}(\theta)\right\rangle=0$.
We now turn to studying the stability of the bifurcating periodic solution. As in $\mathrm{Xu}$ and $\mathrm{Li}$ [30], the bifurcating periodic solution $Z(t, \mu(\varepsilon))$ has amplitude $O(\varepsilon)$ and nonzero Floquet exponent $\beta(\varepsilon)$, with $\beta(0)=0$. Under these assumptions, $\mu$ and $\beta$ are given by

$$
\begin{aligned}
& \mu=\mu_{2} \varepsilon_{2}+\mu_{4} \varepsilon_{4}+\cdots, \\
& \beta=\beta_{2} \varepsilon_{2}+\beta_{4} \varepsilon_{4}+\cdots .
\end{aligned}
$$

The sign of $\mu_{2}$ indicates the direction of the bifurcation, while $\beta_{2}$ determines the stability of $Z(t, \mu(\varepsilon))$, with $Z(t, \mu(\varepsilon))$ being stable if $\beta_{2}<0$ and unstable if $\beta_{2}>0$.

In what follows, we will show how to derive these coefficients (i.e., $\mu_{2}$ and $\beta_{2}$ ). To this effect, we first construct the coordinates needed to describe the center manifold $\Omega_{0}$ near $\mu=0$, which is a local invariant, attracting a twodimensional manifold [30].

Let $u_{t}$ be the solution of (63) when $\mu=0$, and define

$$
\begin{aligned}
z(t) & =\left\langle q^{*}, u_{t}\right\rangle, \\
W(t, \theta) & =u_{t}(t, \theta)-2 \operatorname{Re}(z(t), q(\theta)) .
\end{aligned}
$$

On the center manifold $\Omega_{0}$, we have $W(t, \theta)=W(z(t)$, $\bar{z}(t), \theta)$, where

$$
\begin{aligned}
W(z, \bar{z}, \theta)= & W_{20}(\theta) \frac{z^{2}}{2}+W_{11}(\theta) z \bar{z}+W_{02}(\theta) \frac{\bar{z}^{2}}{2} \\
& +\cdots
\end{aligned}
$$

and $z$ and $\bar{z}$ are local coordinates for the center manifold $\Omega_{0}$ in the direction of $q^{*}$ and $\bar{q}^{*}$. Note that $W$ is real if $u_{t}$ is real (i.e., we consider only the real solutions). For the solution $u_{t} \in \Omega_{0}$, since $\mu=0$, it follows that

$$
\begin{aligned}
\dot{z}_{1}(t)= & \left\langle q^{*}, \dot{u}_{t}\right\rangle=\left\langle q^{*}, A(\mu) u_{t}+R(\mu) u_{t}\right\rangle \\
= & i \omega_{10} \tau_{10} z(t) \\
& +\bar{q}^{*}(0) F(0, W(t, 0)+2 \operatorname{Re}(z(t) q(\theta))) .
\end{aligned}
$$

We can rewrite this equation as

$$
\begin{aligned}
\dot{z}_{1}(t)= & i \omega_{10} \tau_{10} z(t)+g(z(t), \bar{z}(t)), \text { where } \\
g(z(t), \bar{z}(t))= & g_{20} \frac{z^{2}(t)}{2}+g_{11} z(t) \bar{z}(t)+g_{02} \frac{\bar{z}^{2}(t)}{2} \\
& +g_{21} \frac{z^{2}(t) \bar{z}(t)}{2}+\cdots .
\end{aligned}
$$

In Appendix B, we show how to compute the $g_{20}, g_{11}, g_{02}$, and $g_{21}$ coefficients explicitly. Using these coefficients, we can therefore compute

$$
C_{1}(0)=\frac{i}{2 \omega_{10}}\left(g_{20} g_{11}-2\left|g_{11}\right|^{2}-\frac{1}{3}\left|g_{02}\right|\right)+\frac{g_{21}}{2} \text {. }
$$


From (77), it is easy to get the values of $T_{2}, \mu_{2}$, and $\beta_{2}$, as follows:

$$
\begin{aligned}
& \mu_{2}=-\frac{\operatorname{Re}\left(C_{1}(0)\right)}{\operatorname{Re} \lambda^{\prime}\left(\tau_{10}\right)}, \\
& T_{2}=-\frac{\operatorname{Im}\left(C_{1}(0)+\mu_{2} \operatorname{Im}\left(\lambda^{\prime}\left(\tau_{10}\right)\right)\right)}{\omega_{10} \tau_{10}}, \\
& \beta_{2}=2 \operatorname{Re}\left(C_{1}(0)\right) .
\end{aligned}
$$

Using these results, we establish the following theorem on the system trajectories properties.

Theorem 7 (see [14]). If all the conditions above are satisfied (i.e., differential system (2) undergoes a Hopf bifurcation at the positive equilibrium $E_{0}\left(p_{10}, p_{20}\right)$ for $\left.\tau_{1}=\tau_{10}\right)$, then

(i) $\mu=0$ is the Hopf bifurcation value of system (57);

(ii) $\mu_{2}$ establishes the direction of the Hopf bifurcation: if $\mu_{2}>0(<0)$, then the Hopf bifurcation is supercritical (subcritical) and the bifurcating periodic solution exists for $\tau_{1}>\tau_{10}\left(<\tau_{10}\right)$;

(iii) $\beta_{2}$ determines the stability of the bifurcating periodic solutions: if $\beta_{2}<0(>0)$, then the bifurcating periodic solutions are stable (unstable);

(iv) $T_{2}$ determines the period of the bifurcating periodic solutions: the period increases (decreases) if $T_{2}>0(<$ $0)$.

\section{Numerical Illustrations}

To illustrate our results, we employ several numerical simulations. To this purpose, we set $m_{i} \in(0,1)$ and $v_{i}>0$ (with $i=1,2$ ) and choose $q_{1}, q_{2}$ according to Lemma 1 and Proposition 2. Specifically, let $m_{1}=0.2, m_{2}=0.3, v_{1}=$ 20, $v_{2}=10$, and $q_{1}=2.5<m_{1} v_{1}=4$. Using Lemma 1 and Proposition 2, we set $q_{2}=1.525$. From Proposition 2, we then get $a_{3}=0.18, a_{2}=-0.77, a_{1}=-0.251$, and $a_{0}=-2.44$. Using (9), we have that $p_{10}=16.53$ and $p_{20}=11.95$. With this set of parameters, we obtain the following results:

(1) If $\tau_{1}=\tau_{2}=0$, from (20) it follows that $\lambda_{1}=-3.4302$ and $\lambda_{2}=-7.378$. The system is therefore locally asymptotically stable.

(2) If $\tau_{1}=\tau_{2}=\tau$, from (27) it follows that $\omega_{0}=$ 0.6292394611 and from (35) it follows that $\tau_{0}=1.02$. For $\tau<\tau_{0}$, the system is asymptotically stable; for $\tau=\tau_{0}$, it has a Hopf bifurcation; and for $\tau>\tau_{0}$, it is unstable.

The orbits $\left(t, p_{1}(t)\right)$ (in black) and $\left(t, p_{2}(t)\right)$ (in blue) are given in Figures 1, 3, and 5 for $\tau<\tau_{0}, \tau=\tau_{0}$, and $\tau>\tau_{0}$, respectively. Similarly, Figures 2, 4, and 6 plot the market index $(t, I(t))$, where $I(t)=p_{1}(t)-p_{2}(t)$, for each of the three cases.

(3) If $\tau_{1}<\tau_{10}, \tau_{2}=0$; from (39) it follows that $\omega_{10}=$ 12.265. From (42) it follows that $\tau_{10}=0.57185$. For

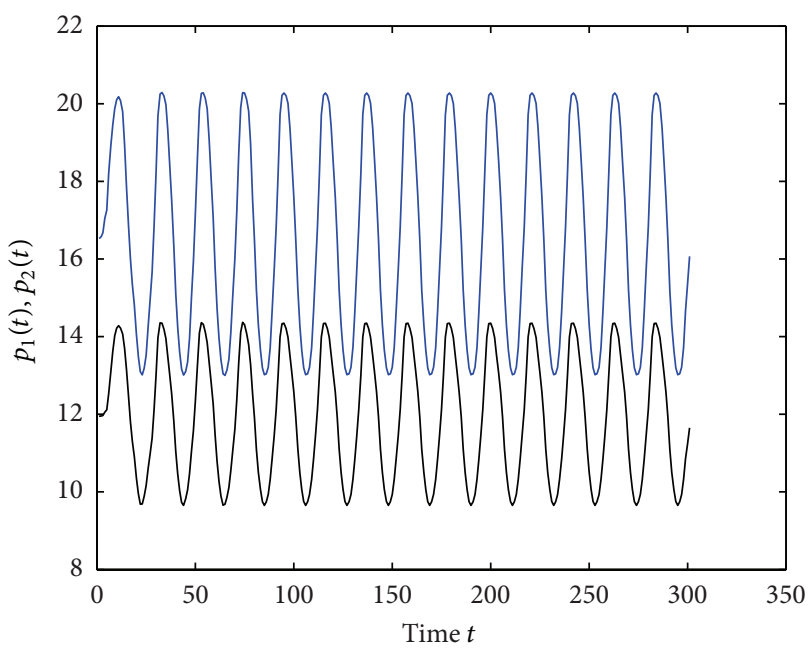

Figure 1: $\left(t, p_{1}(t)\right)$ and $\left(t, p_{2}(t)\right)$, for $\tau<\tau_{0}$.

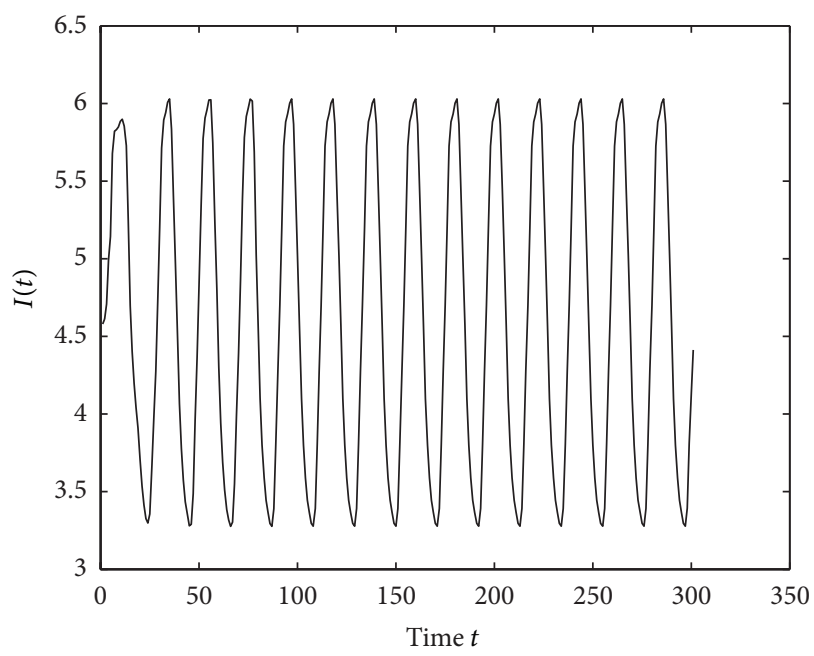

Figure 2: $(t, I(t))$, for $\tau<\tau_{0}$.

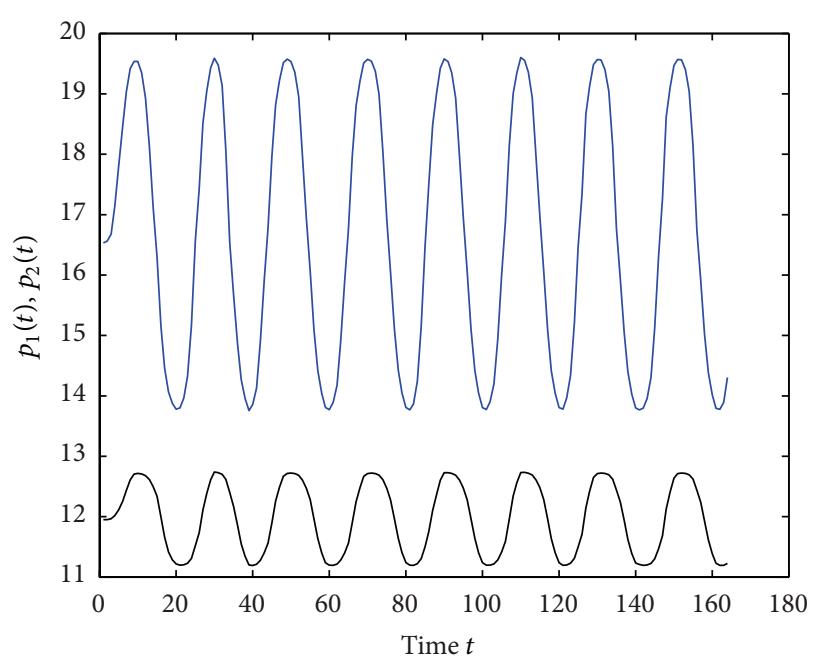

Figure 3: $\left(t, p_{1}(t)\right)$ and $\left(t, p_{2}(t)\right)$, for $\tau=\tau_{0}$. 


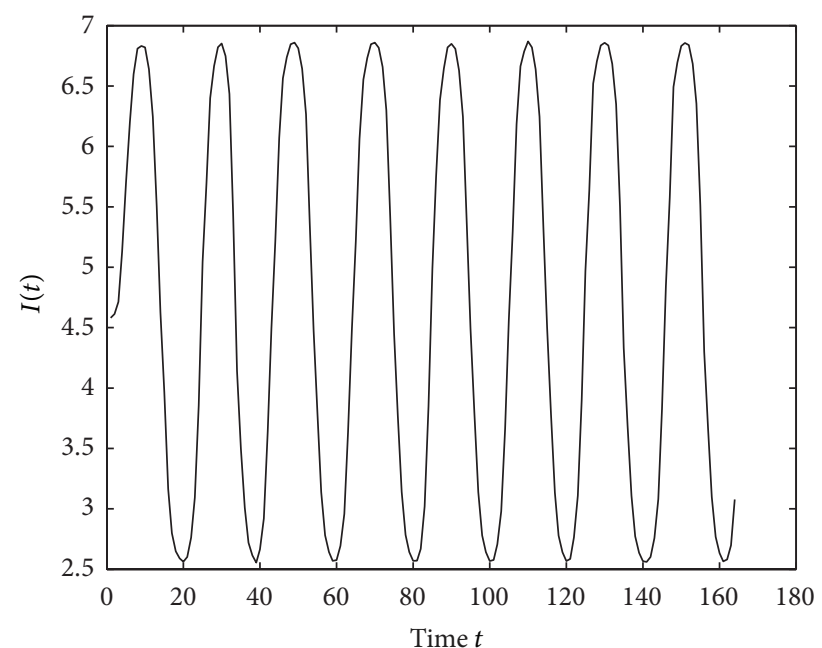

Figure $4:(t, I(t))$, for $\tau=\tau_{0}$.

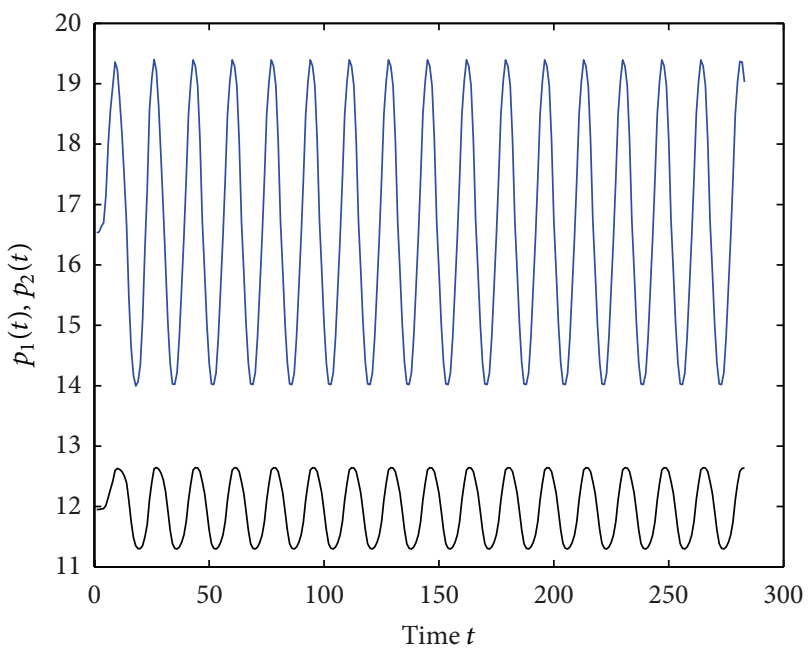

Figure 5: $\left(t, p_{1}(t)\right)$ and $\left(t, p_{2}(t)\right)$, for $\tau>\tau_{0}$.

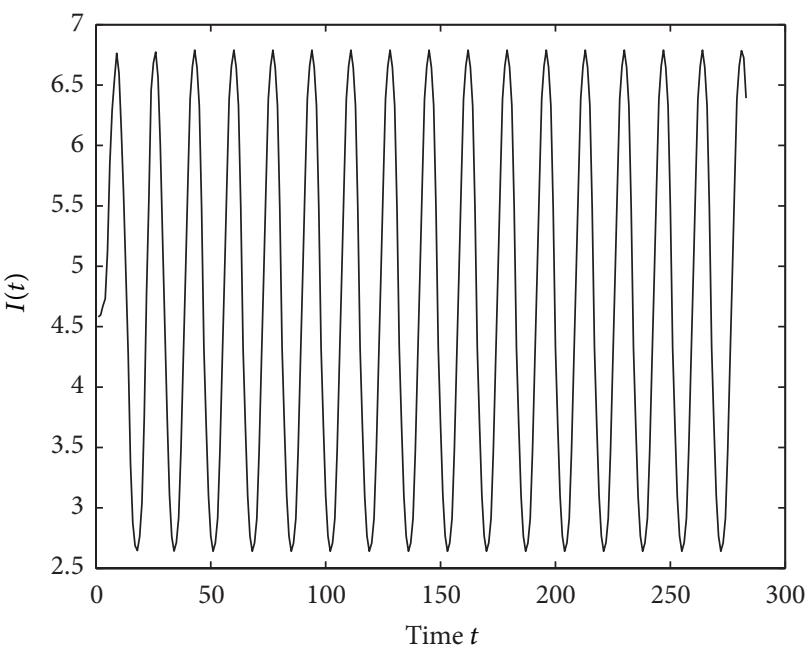

Figure 6: $(t, I(t))$, for $\tau>\tau_{0}$.

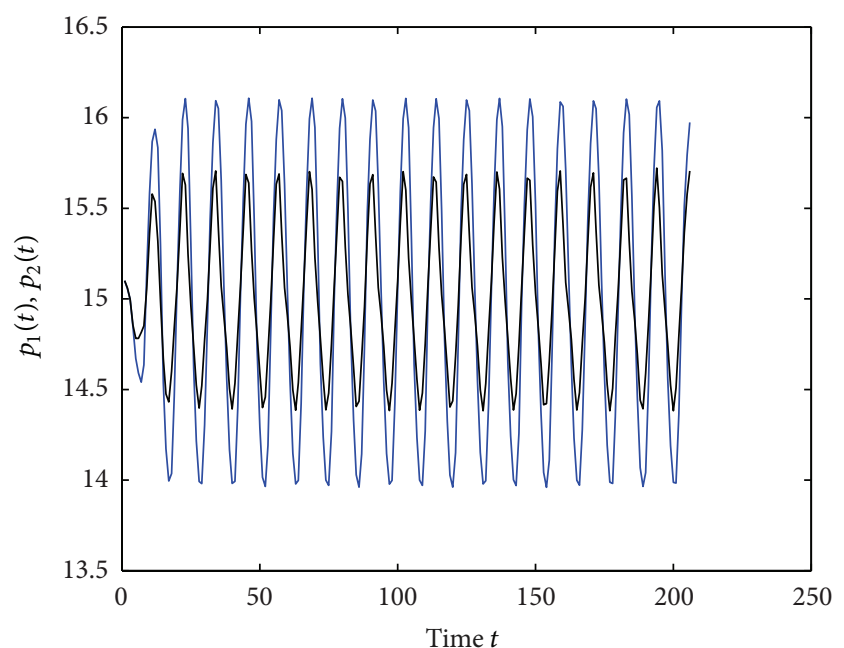

Figure $7:\left(t, p_{1}(t)\right)$ and $\left(t, p_{2}(t)\right)$, for $\tau<\tau_{0}$.

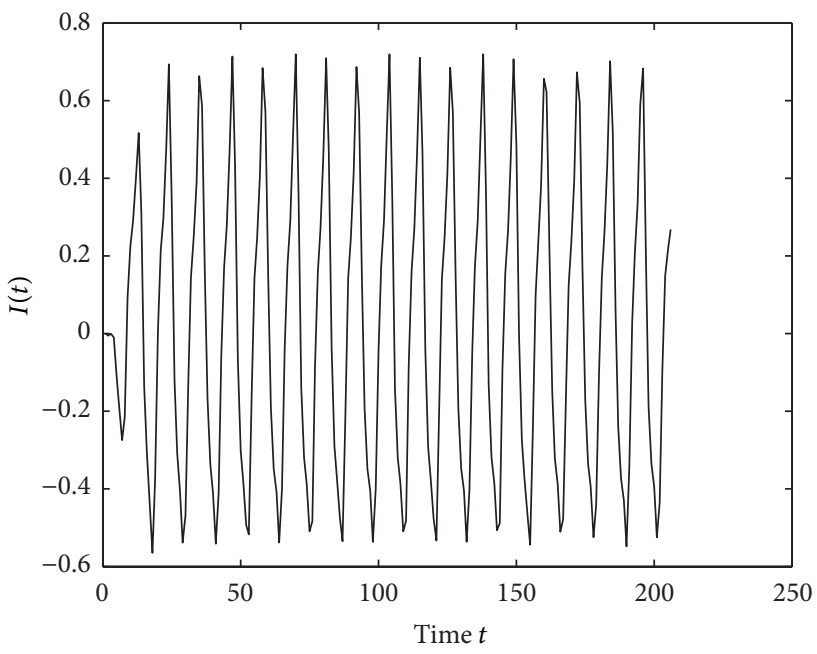

Figure 8: $(t, I(t))$, for $\tau<\tau_{0}$.

$\tau_{1}<\tau_{10}$, the system is locally asymptotically stable. For $\tau_{1}=\tau_{10}$, the system has a Hopf bifurcation. Finally, for $\tau_{1}>\tau_{10}$, the system is unstable.

(4) If $\tau_{1}>0, \tau_{2}>0$, let $\tau_{1}=0.5$. From (50), using the plot of the function $K_{12}(\omega)$, it follows that $\omega_{21}=9.5$, and from (55) we get that $\tau_{21}=0.1981$. For $\tau_{10}=0.5$ and $\tau_{2}<\tau_{21}$, the system is locally asymptotically stable. If $\tau_{2}<\tau_{21}$, the system has a Hopf bifurcation. Finally, if $\tau_{2}>\tau_{21}$, the system is unstable.

The Lyapunov coefficients are $\mu_{2}=2.203985594, T_{2}=$ 8.289687798, and $\beta_{20}=89.44496112$. Hence, the Hopf bifurcation is supercritical and with increasing period, while the bifurcating periodic solutions are unstable.

As before, for $\tau_{2}<\tau_{21}$, the orbits $\left(t, p_{1}(t)\right)$ (in black) and $\left(t, p_{2}(t)\right)$ (in blue) are given in Figure 7 , while Figure 8 plots $(t, I(t))$, where $I(t)=p_{1}(t)-p_{2}(t)$ denotes the market index.

We thus can conclude that all numerical simulations verify our previous theoretical findings. 


\section{Conclusions}

This paper considers a deterministic dynamic model with delay for two financial markets that trade one asset. The deterministic system is analyzed both analytically and numerically. We study two different cases of time delay, namely, when both markets experience the same time delay and when the time delay is different across markets. The common conclusion from these two scenarios is that coupled speculative markets with chartists and fundamentalists in each, but with different dynamics, can be synchronized through diffusive coupling. The stable converging dynamics is replaced with limit cycle oscillations around the fundamental price. This qualitative change in the dynamic behavior shows that coupling and contagion between different markets can indeed lead to transmission of fluctuations across financial markets. Since the delay can lead to instability, the next natural step would be to consider the stochastic approach. We leave this issue for future research.

In terms of contribution, our work ultimately provides a real-world application of bifurcation concepts to a situation widely observed in the financial world. To this effect, we provide a rigorous analysis of the dynamic properties of a model that can successfully explain stylized facts related to financial market behavior. Once established, the same methodology could be easily implemented in economics, demography, and social sciences in general, allowing further literature advances in these fields. For instance, this application fits well and can be quickly integrated in the structural work done in these fields.

\section{Appendices}

\section{A. Data of Lemma A.1}

Lemma A.1 (see [31, Corollary 2.4, page 867]). For the transcendental equation

$$
\begin{aligned}
& \sum_{i=0}^{n} P\left(\lambda, e^{-\lambda \tau_{1}}, \ldots, e^{-\lambda \tau_{n}}\right) \\
& \quad=\lambda^{n}+\sum_{i=0}^{n} P^{i}\left(\lambda, e^{-\lambda \tau_{1}}, \ldots, e^{-\lambda \tau_{n}}\right)=0,
\end{aligned}
$$

where

$$
\begin{aligned}
P^{i} & \left(\lambda, e^{-\lambda \tau_{1}}, \ldots, e^{-\lambda \tau_{n}}\right) \\
=\left[\beta_{1}^{(i)} \lambda^{n-1}+\cdots+\beta_{n-1}^{(i)} \lambda+\beta_{n}^{(i)}\right] e^{-\lambda \tau_{i}} & \\
\quad i & =0,1, \ldots, n,
\end{aligned}
$$

as $\left(\tau_{1}, \ldots, \tau_{n}\right)$ vary, the sum of the orders of the zeros of $P\left(\lambda, e^{-\lambda \tau_{1}}, \ldots, e^{-\lambda \tau_{n}}\right)$ in the open right half plane can change only if a zero appears on or crosses the imaginary axis.

Proof of Proposition 6. (i) Let $q(\theta)$ be given by (66). It follows from above that

$$
A q(0)=i \omega_{10} \tau_{10} q(0)
$$

implying

$$
\tau_{10}\left(\begin{array}{cc}
i \omega_{10} \tau_{10}-a_{11}-b_{11} e^{-i \omega_{10} \tau_{10}} & -a_{12} \\
-a_{21} & i \omega_{10} \tau_{10}-a_{22}-b_{22} e^{-i \omega_{10} \tau_{2} / \tau_{10}}
\end{array}\right)\left(\begin{array}{l}
1 \\
\alpha
\end{array}\right)=\left(\begin{array}{l}
0 \\
0
\end{array}\right) .
$$

As a result, (67) follows.

(ii) Let $q^{*}(s)$ be given by (68). From

$$
A^{*} q^{*}(0)=-i \omega_{10} \tau_{10} q^{*}(0),
$$

it follows that $\alpha^{*}$ is given by (70).

\section{B. Coefficients of Center Manifold}

Following Hassard et al. [14], by substituting (63) in (75) we have

$$
\dot{W}=\dot{u}_{t}-\dot{z} q-\dot{\bar{z}} \bar{q}
$$

Hence,

$$
\begin{aligned}
\dot{W} & =A W-2 \operatorname{Re}\left[\bar{q}^{*}(0) F(z, \bar{z}) q(\theta)\right]+R u_{t} \\
& = \begin{cases}A W-2 \operatorname{Re}\left[\bar{q}^{*}(0) F(z, \bar{z}) q(\theta)\right], & \text { if } \theta \in[-1,0), \text { if } \theta=[-1,0) \text { def } \\
A W-2 \operatorname{Re}\left[\bar{q}^{*}(0) F(z, \bar{z}) q(0)\right]+\operatorname{Re}(w(0)+2 \operatorname{Re}(z(t) q(0))), & \text { if } \theta=0\end{cases} \\
& +H(z, \bar{z}, \theta),
\end{aligned}
$$

where

$$
\begin{aligned}
H(z, \bar{z}, \theta)= & h_{20}(\theta) \frac{z^{2}(t)}{2}+h_{11}(\theta) z(t) \bar{z}(t) \\
& +h_{02}(\theta) \frac{\bar{z}^{2}(t)}{2}+\cdots
\end{aligned}
$$

Taking the derivative of $W$ with respect to $t$ in (74), we have

$$
\dot{W}=W_{z} \dot{z}+W_{\bar{z}} \dot{\bar{z}}
$$


From (74), (76), and (B.4), we obtain

$$
\begin{aligned}
\dot{W}= & \left(W_{20} z+W_{11} \bar{z}+\cdots\right)\left(i \omega_{10} \tau_{10} z+g\right) \\
& +\left(W_{11} z+W_{02} \bar{z}+\cdots\right)\left(-i \omega_{10} \tau_{10} \bar{z}+\bar{g}\right),
\end{aligned}
$$

and substituting (74), (B.3), and (B.2) yields

$$
\begin{aligned}
\dot{W}= & \left(A W_{20}+h_{20}\right) \frac{z^{2}}{2}+\left(A W_{11}+h_{11}\right) z \bar{z} \\
& +\left(A W_{02}+h_{02}\right) \frac{\bar{z}^{2}}{2}+\cdots
\end{aligned}
$$

Comparing the coefficients of (B.5) and (B.6), the following equalities hold:

$$
\begin{aligned}
\left(A-2 i \omega_{10} \tau_{10} I\right) W_{20}(t) & =-h_{20}(\theta), \\
A W_{11}(t) & =-h_{11}(\theta),
\end{aligned}
$$

where $I$ is a $2 \times 2$ unity matrix.

From (75) and (76), we obtain

$$
\begin{aligned}
& g(z(t), \bar{z}(t))=\bar{q}^{*} F(z, \bar{z}) \\
& =\frac{\tau_{10}}{\bar{\rho}}\left(1, \bar{\alpha}^{*}\right)\left(\begin{array}{c}
F_{1}\left(u_{1 t}(0), u_{1 t}(-1)\right) \\
F_{2}\left(u_{2 t}(0), u_{2 t}\left(-\frac{\tau_{2}}{\tau_{10}}\right)\right)
\end{array}\right),
\end{aligned}
$$

where

$$
\begin{aligned}
u_{t}= & \left(u_{1 t}(\theta), u_{2 t}(\theta)\right)^{T} \\
= & W(t, \theta)+z q(\theta)+\bar{z} \bar{q}(\theta), \\
q(\theta)= & (1, \alpha) e^{i \omega_{10} \tau_{10} \theta}, \\
u_{1 t}(0)= & z+\bar{z}+W_{20}^{(1)}(0) \frac{z^{2}}{2}+W_{11}^{(1)}(0) z \bar{z} \\
& +W_{02}^{(1)}(0) \frac{\bar{z}^{2}}{2}+\cdots \\
u_{2 t}(0)= & \alpha z+\bar{\alpha} \bar{z}+W_{20}^{(2)}(0) \frac{z^{2}}{2}+W_{11}^{(2)}(0) z \bar{z} \\
& +W_{02}^{(2)}(0) \frac{\bar{z}^{2}}{2}+\cdots, \\
u_{1 t}(-1)= & z e^{-i \omega_{10} \tau_{10}}+\bar{z} e^{i \omega_{10} \tau_{10}}+W_{20}^{(1)}(-1) \frac{z^{2}}{2} \\
& +W_{11}^{(1)}(-1) z \bar{z}+W_{02}^{(1)}(-1) \frac{\bar{z}^{2}}{2} \\
& +\cdots,
\end{aligned}
$$

$$
\begin{aligned}
u_{2 t}\left(-\frac{\tau_{2}}{\tau_{10}}\right)= & \alpha z e^{-i \omega_{10} \tau_{2} / \tau_{10}} \\
& +\bar{\alpha} z e^{i \omega_{10} \tau_{2} / \tau_{10}} W_{20}^{(2)}\left(-\frac{\tau_{2}}{\tau_{10}}\right) \frac{z^{2}}{2} \\
& +W_{11}^{(2)}\left(-\frac{\tau_{2}}{\tau_{10}}\right) z \bar{z} \\
& +W_{02}^{(2)}\left(-\frac{\tau_{2}}{\tau_{10}}\right) \frac{\bar{z}^{2}}{2}+\cdots
\end{aligned}
$$

Proposition B.1. The coefficients $g_{20}, g_{11}, g_{02}$, and $g_{21}$ are given by

$$
\begin{aligned}
& g_{20}=\frac{2 \tau_{10}}{\bar{\rho}}\left(l_{1}+l_{2} e^{-i \omega_{10} \tau_{10}}+\alpha^{2} \bar{\alpha}^{*}\left(k_{1}\right.\right. \\
& \left.\left.+k_{2} e^{-i \omega_{10} \tau_{2} / \tau_{10}}\right)\right) \text {, } \\
& g_{11}=\frac{\tau_{10}}{\bar{\rho}}\left(l_{1}+l_{2}\left(e^{i \omega_{10} \tau_{10}}+e^{-i \omega_{10} \tau_{10}}\right)+\bar{\alpha}^{2} \alpha^{*}\left(k_{1}\right.\right. \\
& \left.\left.+k_{2}\left(e^{i \omega_{10} \tau_{2} / \tau_{10}}+e^{-i \omega_{10} \tau_{2} / \tau_{10}}\right)\right)\right) \text {, } \\
& g_{02}=\frac{2 \tau_{10}}{\bar{\rho}}\left(l_{1}+l_{2} e^{i \omega_{10} \tau_{10}}+\alpha^{2} \bar{\alpha}^{*}\left(k_{1}+k_{2} e^{i \omega_{10} \tau_{2} / \tau_{10}}\right)\right) \text {, } \\
& g_{21}=\frac{2 \tau_{10}}{\bar{\rho}}\left(l_{1}\left(2 W_{11}^{(1)}(0)+W_{20}^{(1)}(0)\right)\right. \\
& +l_{2}\left(W_{11}^{(1)}(0) e^{-i \omega_{10} \tau_{10}}+\frac{1}{2} W_{20}^{(1)}(0) e^{i \omega_{10} \tau_{10}}\right. \\
& \left.+W_{11}^{(1)}(-1)+\frac{1}{2} W_{20}^{(1)}(-1)\right)+3 l_{3}+l_{4}\left(e^{i \omega_{10} \tau_{10}}\right. \\
& \left.\left.+2 e^{-i \omega_{10} \tau_{10}}\right)\right)+l_{5}\left(2+e^{-2 i \omega_{10} \tau_{10}}\right)+3 l_{6} e^{-2 i \omega_{10} \tau_{10}} \\
& +\bar{\alpha}^{*}\left(k_{1}\left(2 \alpha W_{11}^{(2)}(0)+\bar{\alpha} W_{20}^{(2)}(0)\right)\right. \\
& +k_{2}\left(\alpha W_{11}^{(2)}\left(-\frac{\tau_{2}}{\tau_{10}}\right)+\alpha W_{11}^{(2)}(0) e^{-i \omega_{10} \tau_{2} / \tau_{10}}\right. \\
& \left.+\frac{\alpha}{2} W_{11}^{(2)}(0) e^{-i \omega_{10} \tau_{2} / \tau_{10}}+\alpha \bar{\alpha} W_{20}^{(2)}\left(-\frac{\tau_{2}}{\tau_{10}}\right)\right) \\
& +3 k_{3} \alpha^{2} \bar{\alpha}+k_{4} \alpha^{2} \bar{\alpha}\left(e^{i \omega_{10} \tau_{2} / \tau_{10}}+2 e^{-i \omega_{10} \tau_{2} / \tau_{10}}\right) \\
& \left.+k_{5} \alpha^{2} \bar{\alpha}\left(2+e^{-i \omega_{10} \tau_{2} / \tau_{10}}\right)+3 k_{6} \alpha^{2} \bar{\alpha} e^{-i \omega_{10} \tau_{2} / \tau_{10}}\right) .
\end{aligned}
$$

Proof. Comparing the coefficients of (77) and (B.9), (B.10) follows. 
Proposition B.2. The vectors, $W_{11}(\theta)$ and $W_{20}(\theta)$, are given by

$$
\begin{aligned}
W_{11}(\theta)= & -\frac{i g_{11}}{\omega_{10} \tau_{10}} q(0) e^{i \omega_{10} \tau_{10} \theta} \\
& -\frac{\bar{g}_{11}}{i \omega_{10} \tau_{10}} \bar{q}(0) e^{-i \omega_{10} \tau_{10} \theta}+E_{1},
\end{aligned}
$$

$$
-\frac{i \bar{g}_{02}}{3 \omega_{10} \tau_{10}} \bar{q}(0) e^{-i \omega_{10} \tau_{10} \theta}+E_{2} e^{2 i \omega_{10} \tau_{10} \theta}
$$

where

$$
\begin{aligned}
& E_{2}=\left(\begin{array}{cc}
2 i \omega_{10} \tau_{10}-a_{11}-b_{11} e^{-2 i \omega_{10} \tau_{10}} & -a_{12} \\
-a_{21} & 2 i \omega_{10} \tau_{10}-a_{22}-b_{22} e^{-2 i \omega_{10} \tau_{2} / \tau_{10}}
\end{array}\right)^{-1}\left(\begin{array}{c}
2 l_{1}+2 l_{2} e^{-i \omega_{10} \tau_{10}} \\
2 \alpha^{2}\left(k_{1}+k_{2} e^{-i \omega_{10} \tau_{2} / \tau_{10}}\right)
\end{array}\right), \\
& E_{1}=\left(\begin{array}{cc}
-a_{11}-b_{11} & -a_{12} \\
-a_{21} & -a_{22}-b_{22}
\end{array}\right)^{-1}\left(\begin{array}{c}
l_{1}+l_{2}\left(e^{i \omega_{10} \tau_{10}}+e^{-i \omega_{10} \tau_{10}}\right) \\
\alpha \bar{\alpha}\left(k_{1}+k_{2}\left(e^{i \omega_{10} \tau_{2} / \tau_{10}}+e^{-i \omega_{10} \tau_{2} / \tau_{10}}\right)\right)
\end{array}\right) .
\end{aligned}
$$

Proof. First, we use (B.2) to obtain

$$
\begin{aligned}
H(z, \bar{z}, \theta)=-2 \operatorname{Re}\left[\bar{q}^{*}(0) F(z, \bar{z}) q(\theta)\right]+R u_{t} \\
=-g q(\theta)-\bar{g} \bar{q}(\theta)+R u_{t} \\
=-\left(\frac{1}{2} g_{20} z^{2}+g_{11} z \bar{z}+\frac{1}{2} g_{02} \bar{z}^{2}+\cdots\right) q(\theta) \\
\quad-\left(\frac{1}{2} \bar{g}_{20} z^{2}+\bar{g}_{11} z \bar{z}+\frac{1}{2} \bar{g}_{02} \bar{z}^{2}+\cdots\right) \bar{q}(\theta) \\
\quad+R u_{t} .
\end{aligned}
$$

Comparing the coefficients with (B.3), for $\theta \in(-1,0]$, we obtain

$$
\begin{aligned}
& h_{20}(\theta)=-g_{20} q(\theta)-\bar{g}_{20} \bar{q}(\theta), \\
& h_{11}(\theta)=-g_{11} q(\theta)-\bar{g}_{11} \bar{q}(\theta) .
\end{aligned}
$$

Substituting the above equalities into (B.7) and (B.8), respectively, and with $A=A(0)$ being given by (62), we get that

$$
\begin{aligned}
& \dot{W}_{20}(\theta)=2 i \omega_{10} \tau_{10} W_{20}(\theta)+g_{20} q(\theta)+\bar{g}_{20} \bar{q}(\theta), \\
& \dot{W}_{11}(\theta)=g_{11} q(\theta)+\bar{g}_{11} \bar{q}(\theta) .
\end{aligned}
$$

Solving (B.16) leads to (B.12). To obtain the appropriate $E_{1}$ and $E_{2}$, we proceed as follows.

From (B.7) and (B.8), it follows that

$$
\begin{aligned}
& A W_{20}(0)=2 i \omega_{10} \tau_{10} W_{20}(0)-h_{20}(0), \\
& A W_{11}(0)=-h_{11}(0) .
\end{aligned}
$$

From the definition of $A$ in (62), we obtain

$$
\begin{aligned}
& \int_{-1}^{0} d \eta(\theta) W_{20}(\theta)=2 i \omega_{10} \tau_{10} W_{20}(0)-h_{20}(0), \\
& \int_{-1}^{0} d \eta(\theta) W_{11}(\theta)=-h_{11}(0) .
\end{aligned}
$$

From (B.15) and the definition of $F(\mu, \phi)$, we have that

$$
\begin{aligned}
& h_{20}(0) \\
& =-g_{20} q(\theta)-\bar{g}_{20} \bar{q}(\theta) \\
& +\tau_{0}\left(\begin{array}{c}
2 l_{1}+2 l_{2} e^{-i \omega_{10} \tau_{10}} \\
2 \alpha^{2}\left(k_{1}+k_{2} e^{-i \omega_{10} \tau_{2} / \tau_{10}}\right)
\end{array}\right), \\
& h_{11}(0) \\
& =-g_{11} q(\theta)-\bar{g}_{11} \bar{q}(\theta) \\
& +\tau_{0}\left(\begin{array}{c}
l_{1}+l_{2}\left(e^{i \omega_{10} \tau_{10}}+e^{-i \omega_{10} \tau_{10}}\right) \\
\alpha \bar{\alpha}\left(k_{1}+k_{2}\left(e^{i \omega_{10} \tau_{2} / \tau_{10}}+e^{-i \omega_{10} \tau_{2} / \tau_{10}}\right)\right)
\end{array}\right) .
\end{aligned}
$$

Substituting (B.12) and (B.19) into (B.18) and using the fact that

$$
\begin{gathered}
\left(i \omega_{10} \tau_{10} I-\int_{-1}^{0} e^{i \omega_{10} \tau_{10} \theta} d \eta(\theta)\right) q(0)=0, \\
\left(-i \omega_{10} \tau_{10} I-\int_{-1}^{0} e^{-i \omega_{10} \tau_{10} \theta} d \eta(\theta)\right) \bar{q}(0)=0,
\end{gathered}
$$

we obtain

$$
\begin{gathered}
\left(2 i \omega_{10} \tau_{10} I-\int_{-1}^{0} e^{2 i \omega_{10} \tau_{10} \theta} d \eta(\theta)\right) E_{2} \\
=\tau_{0}\left(\begin{array}{c}
2 l_{1}+2 l_{2} e^{-i \omega_{10} \tau_{10}} \\
2 \alpha^{2}\left(k_{1}+k_{2} e^{-i \omega_{10} \tau_{2} / \tau_{10}}\right)
\end{array}\right),
\end{gathered}
$$


which leads to

$$
\left(\begin{array}{cc}
2 i \omega_{10} \tau_{10}-a_{11}-b_{11} e^{-2 i \omega_{10} \tau_{10}} & -a_{12} \\
-a_{21} & 2 i \omega_{10} \tau_{10}-a_{22}-b_{22} e^{-2 i \omega_{10} \tau_{2} / \tau_{10}}
\end{array}\right) E_{2}=\left(\begin{array}{c}
2 l_{1}+2 l_{2} e^{-i \omega_{10} \tau_{10}} \\
2 \alpha^{2}\left(k_{1}+k_{2} e^{-i \omega_{10} \tau_{2} / \tau_{10}}\right)
\end{array}\right),
$$

and so

$$
E_{2}=\left(\begin{array}{cc}
2 i \omega_{10} \tau_{10}-a_{11}-b_{11} e^{-2 i \omega_{10} \tau_{10}} & -a_{12} \\
-a_{21} & 2 i \omega_{10} \tau_{10}-a_{22}-b_{22} e^{-2 i \omega_{10} \tau_{2} / \tau_{10}}
\end{array}\right)^{-1}\left(\begin{array}{c}
2 l_{1}+2 l_{2} e^{-i \omega_{10} \tau_{10}} \\
2 \alpha^{2}\left(k_{1}+k_{2} e^{-i \omega_{10} \tau_{2} / \tau_{10}}\right)
\end{array}\right) .
$$

Similarly, substituting (B.12) and (B.19) into (B.17), we have that

$$
\begin{aligned}
E_{1}= & \left(\begin{array}{cc}
-a_{11}-b_{11} & -a_{12} \\
-a_{21} & -a_{22}-b_{22}
\end{array}\right)^{-1} \\
& \cdot\left(\begin{array}{c}
l_{1}+l_{2}\left(e^{i \omega_{10} \tau_{10}}+e^{-i \omega_{10} \tau_{10}}\right) \\
\alpha \bar{\alpha}\left(k_{1}+k_{2}\left(e^{i \omega_{10} \tau_{2} / \tau_{10}}+e^{-i \omega_{10} \tau_{2} / \tau_{10}}\right)\right)
\end{array}\right) .
\end{aligned}
$$

We can therefore derive $W_{20}(\theta)$ and $W_{11}(\theta)$, with $g_{i j}$ in (B.10) being determined by the parameters and the time delays in system (2).

\section{Conflict of Interests}

The authors declare that there is no conflict of interests regarding the publication of this paper.

\section{Acknowledgment}

The authors are especially grateful to D. Opris, the editor, for helpful comments and suggestions.

\section{References}

[1] Y. Qu and J. Wei, "Global Hopf bifurcation analysis for a timedelayed model of asset prices," Discrete Dynamics in Nature and Society, vol. 2010, Article ID 432821, 17 pages, 2010.

[2] C. Chiarella, R. Dieci, and L. Gardini, "Speculative behaviour and complex asset price dynamics: a global analysis," Journal of Economic Behavior and Organization, vol. 49, no. 2, pp. 173-197, 2002.

[3] C. Chiarella, R. Dieci, and X. He, "Heterogeneity, market mechanisms and asset price dynamics," in Handbook of Financial Markets: Dynamics and Evolution, vol. 5, pp. 277-344, Elsevier, Philadelphia, Pa, USA, 2009.

[4] C. Chiarella and X.-Z. He, "Heterogeneous beliefs, risk, and learning in a simple asset-pricing model with a market maker," Macroeconomic Dynamics, vol. 7, no. 4, pp. 503-536, 2003.

[5] G. Dibeh, "Speculative dynamics in a time-delay model of asset prices,” Physica A, vol. 355, no. 1, pp. 199-208, 2005.
[6] W. A. Brock, C. H. Hommes, and F. O. O. Wagener, "Evolutionary dynamics in markets with many trader types," Journal of Mathematical Economics, vol. 41, no. 1-2, pp. 7-42, 2005.

[7] C. H. Hommes, "Financial markets as nonlinear adaptive evolutionary systems," Quantitative Finance, vol. 1, no. 1, pp. 149-167, 2001.

[8] C. H. Hommes, "Heterogeneous agent models in economics and finance," in Handbook of Computational Economics, vol. 2, no. 23, pp. 1109-1186, 2006.

[9] T. Lux, "The socio-economic dynamics of speculative markets: interacting agents, chaos, and the fat tails of return distributions," Journal of Economic Behavior and Organization, vol. 33, no. 2, pp. 143-165, 1998.

[10] G. Mircea, M. Neamtu, and D. Opris, Uncertain, Stochastic and Fractional Dynamic Systems with Delay. Applications, Lambert Academic Publishing, Saarbrücken, Germany, 2011.

[11] M. Neamtu, G. Mircea, and D. Opris, "Deterministic and stochastic model for the analysis of the asset price," in Proceedings of the 13th International Conference on Mathematics and Computers in Business \& Economics, pp. 165-170, 2012.

[12] R. Sethi, "Endogenous regime switching in speculative markets," Structural Change and Economic Dynamics, vol. 7, no. 1, pp. 99-118, 1996.

[13] J. P. Bouchaud and M. Potters, Theory of Financial Risk and Derivative Pricing: From Statistical Physics to Risk Management, Cambridge University Press, Cambridge, UK, 2nd edition, 2003.

[14] B. D. Hassard, N. D. Kazarinoff, and Y. H. Wan, Theory and Applications of Hopf Bifurcation, vol. 41 of London Mathematical Society Lecture Note, Cambridge University Press, Cambridge, UK, 1981

[15] E. Karaoglu and H. Merdan, "Hopf bifurcations of a ratiodependent predator-prey model involving two discrete maturation time delays," Chaos, Solitons \& Fractals, vol. 68, pp. 159-168, 2014.

[16] L.-Y. Zhang, "Hopf bifurcation analysis in a Monod-Haldane predator-prey model with delays and diffusion," Applied Mathematical Modelling, vol. 39, no. 3-4, pp. 1369-1382, 2015.

[17] C. Chiarella, R. Dieci, and L. Gardini, "The dynamic interaction of speculation and diversification," Applied Mathematical Finance, vol. 12, no. 1, pp. 17-52, 2005. 
[18] G. Dibeh, "Contagion effects in a chartist-fundamentalist model with time delays," Physica A, vol. 382 , no. 1, pp. 52-57, 2007.

[19] D. J. Fenn, M. A. Porter, S. Williams, M. McDonald, N. F. Johnson, and N. S. Jones, "Temporal evolution of financialmarket correlations," Physical Review E, vol. 84, no. 2, Article ID 026109, 2011.

[20] L. H. Ederington and J. H. Lee, "How markets process information: news releases and volatility," The Journal of Finance, vol. 48, no. 4, pp. 1161-1191, 1993.

[21] P. Balduzzi, E. J. Elton, and T. C. Green, "Economic news and bond prices: evidence from the U.S. treasury market," Journal of Financial and Quantitative Analysis, vol. 36, no. 4, pp. 523-543, 2001.

[22] T. G. Andersen, T. Bollerslev, F. X. Diebold, and C. Vega, "Realtime price discovery in global stock, bond and foreign exchange markets," Journal of International Economics, vol. 73, no. 2, pp. 251-277, 2007.

[23] D. J. Fenn, M. A. Porter, M. McDonald, S. Williams, N. F. Johnson, and N. S. Jones, "Dynamic communities in multichannel data: an application to the foreign exchange market during the 2007-2008 credit crisis," Chaos, vol. 19, no. 3, Article ID 033119, 2009.

[24] F. Allen and A. Babus, The Network Challenge: Strategy, Profit, and Risk in an Inter linked World, Wharton School, Philadelphia, Pa, USA, 2009.

[25] R. M. May, S. A. Levin, and G. Sugihara, "Complex systems: ecology for bankers,” Nature, vol. 451, no. 7181, pp. 893-895, 2008.

[26] P. Gai and S. Kapadia, "Contagion in financial networks," Proceedings of the Royal Society A, vol. 466, no. 2120, pp. 24012423, 2010.

[27] T. D. Frank, “Time-dependent solutions for stochastic systems with delays: perturbation theory and applications to financial physics," Physics Letters A, vol. 357, no. 4-5, pp. 275-283, 2006.

[28] B. LeBaron, "Evolution and time horizons in an agent-based stock market," Macroeconomic Dynamics, vol. 5, no. 2, pp. 225254, 2001.

[29] J. K. Hale and S. V. Lunel, Introduction to Functional Differential Equations, Springer, New York, NY, USA, 1993.

[30] C. Xu and P. Li, "Dynamical analysis in a delayed predatorprey model with two delays," Discrete Dynamics in Nature and Society, vol. 2012, Article ID 652947, 22 pages, 2012.

[31] S. Ruan and J. Wei, "On the zeros of transcendental functions with applications to stability of delay differential equations with two delays," Dynamics of Continuous, Discrete and Impulsive Systems Series A: Mathematical Analysis, vol. 10, no. 6, pp. 863874, 2003. 


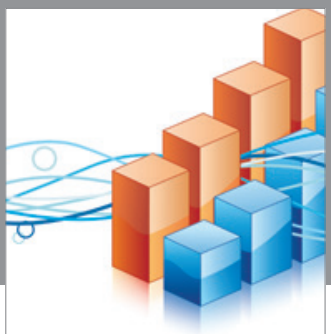

Advances in

Operations Research

vatem alat4

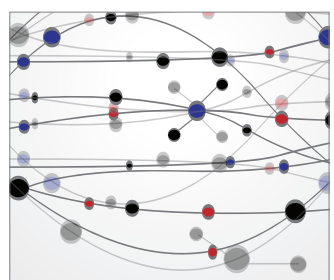

\section{The Scientific} World Journal
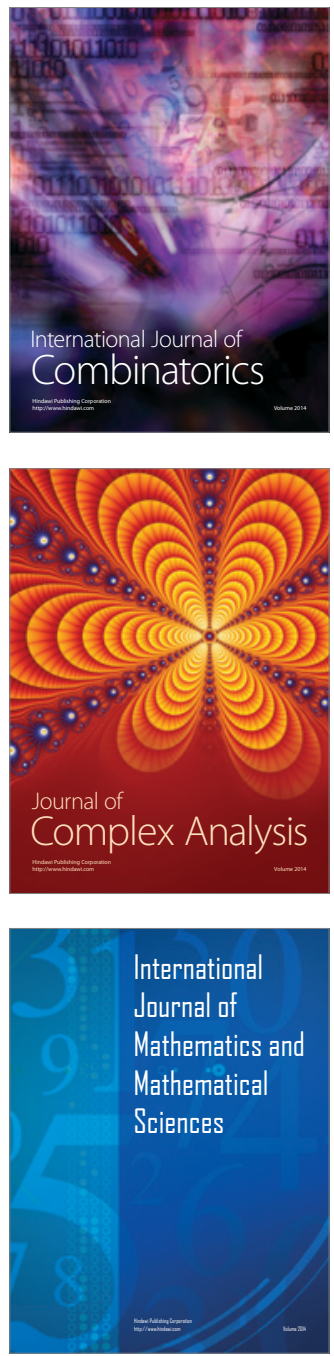
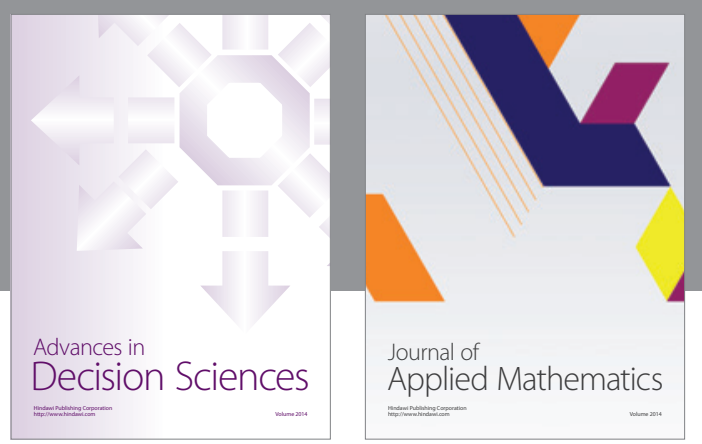

Algebra

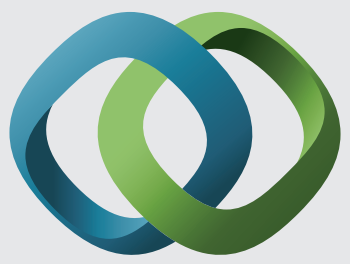

\section{Hindawi}

Submit your manuscripts at

http://www.hindawi.com
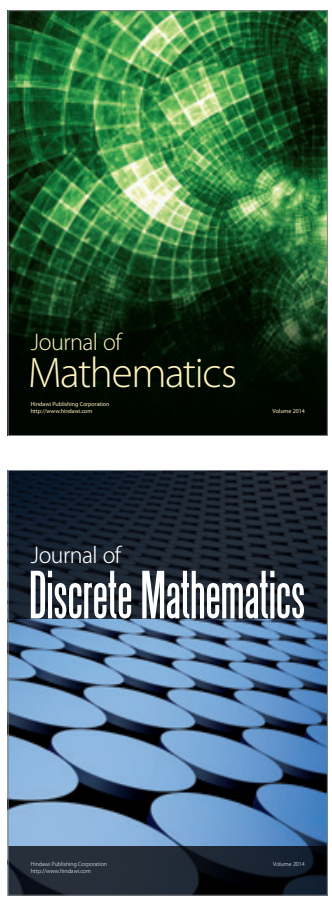

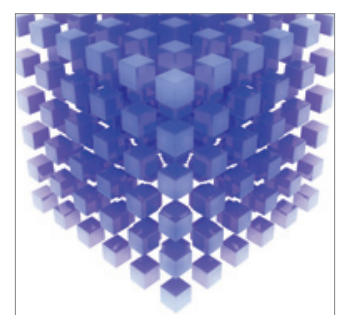

Mathematical Problems in Engineering
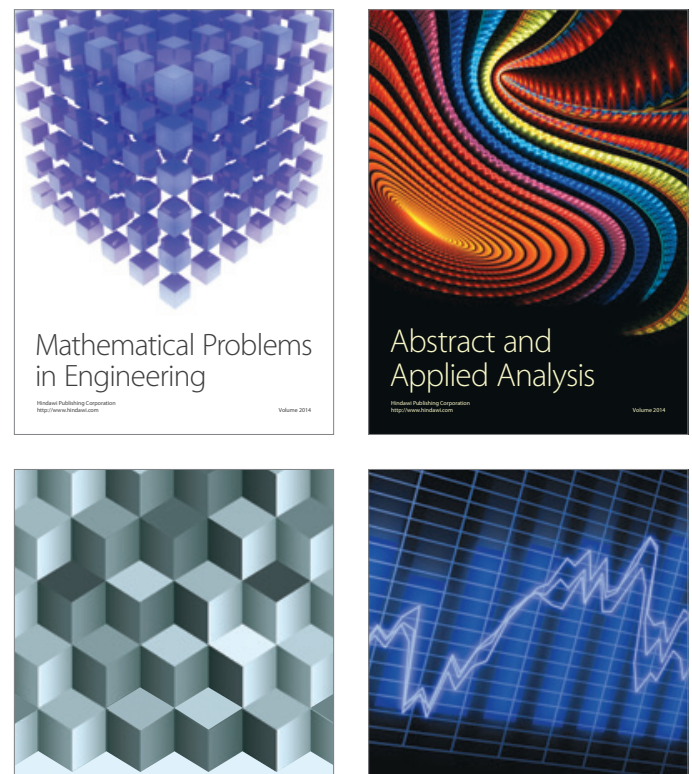

Journal of

Function Spaces

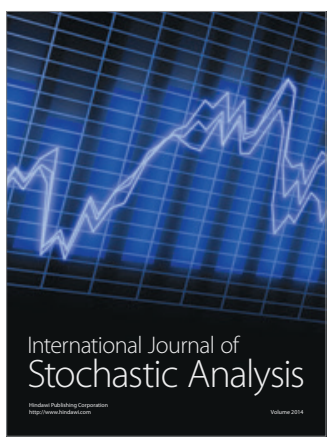

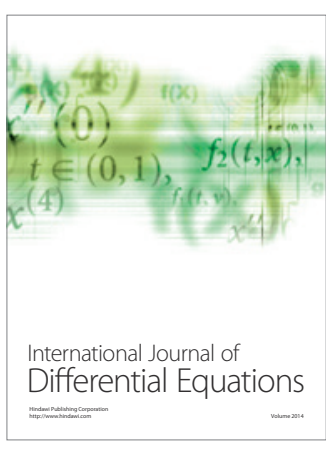
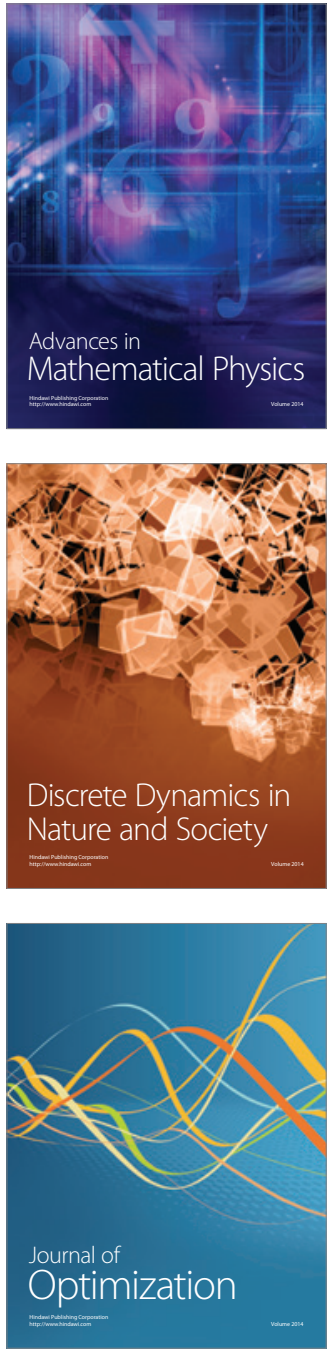